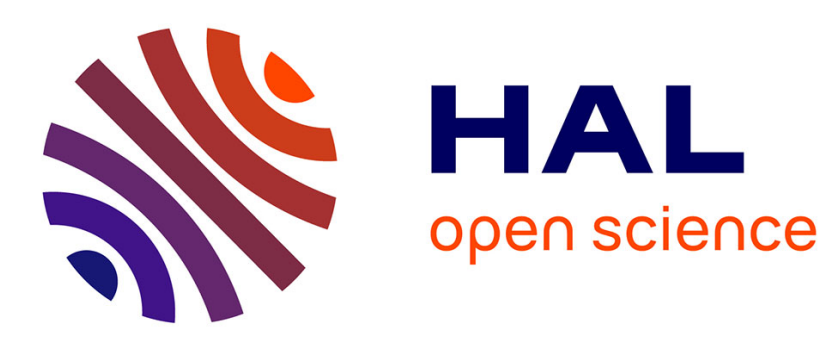

\title{
Recover Dynamic Utility from Observable Process: Application to the economic equilibrium
}

\author{
Nicole El Karoui, Mohamed Mrad
}

\section{To cite this version:}

Nicole El Karoui, Mohamed Mrad. Recover Dynamic Utility from Observable Process: Application to the economic equilibrium. SIAM Journal on Financial Mathematics, 2021. hal-01966312v4

\section{HAL Id: hal-01966312 \\ https://hal.science/hal-01966312v4}

Submitted on 26 Nov 2020

HAL is a multi-disciplinary open access archive for the deposit and dissemination of scientific research documents, whether they are published or not. The documents may come from teaching and research institutions in France or abroad, or from public or private research centers.
L'archive ouverte pluridisciplinaire HAL, est destinée au dépôt et à la diffusion de documents scientifiques de niveau recherche, publiés ou non, émanant des établissements d'enseignement et de recherche français ou étrangers, des laboratoires publics ou privés. 


\title{
Recover Dynamic Utility from Observable Process: Application to the economic equilibrium. ${ }^{* \dagger}$
}

\author{
El Karoui Nicole, ${ }^{\ddagger} \quad$ Mrad Mohamed §
}

\begin{abstract}
Decision making under uncertainty is generally considered as the selection of an optimal sequence of actions in an uncertain environment. Its calibration raises the "inverse" problem to recover the criterium from the data. A classical example in economy is the theory of "revealed preference" introduced by Samuelson in the 40's, Sam38. The observable at a given date $t, x_{t}(x)$, is an increasing function of a real parameter $x$ ( the wealth in economy). The process $\left\{X_{t}(x)\right\}$ is called the characteristic process. The objective is to recover a dynamic stochastic utility $\{U(t, z)\}$, "revealed" in the sense where its performance is without bias, more formally when " $\left\{U\left(t, X_{t}(x)\right)\right\}$ is a martingale". The increasing of $X_{t}(x)$ in $x$, and the concavity of the utility leads to privilege the so-called adjoint process $Y_{t}\left(u_{z}(x)\right):=U_{z}\left(t, X_{t}(x)\right)$ in the linearization of the problem; the one to one correspondence between $U_{z}(t, z)$ and $Y_{t}\left(u_{z}(x)\right)$ for a given characteristic process is used intensively.

We focus on the $(u, X, Y)$ triplets, bringing great attention to their initial conditions. We establish a necessary and sufficient condition for the existence of at least one solution to the "revealed" utility problem. An operational version is as follows: " $\left\{X_{t}(x) Y_{t}(y)\right\}$ is a supermartingale for any $(x, y)$ and $\left\{X_{t}(x) Y_{t}\left(u_{z}(x)\right)\right\}$ is a martingale for $y=u_{z}(x)$ ". Moreover, there is an equivalent intrinsic framework, where in addition the processes " $\left\{X_{t}(x)\right\},\left\{Y_{t}(y)\right\},\{U(t, z)\}$ " are supermartingales.

Itô's semimartingale framework is used to illustrate this characterization. The operational version ensures that the revealed utility is solution of a non-linear SPDE. Less obvious is its interpretation as stochastic value function of some optimization problem. Financial markets framework appears as a special case, under stronger assumptions. Then, we revisit the dynamic equilibrium problem as in $\mathrm{He}$ and Leland [HL93, by considering it as a revealed utility problem. We solve the problem in random environment, by characterizing all the equilibria, in showing that the only possible conjugate dynamic utilities are the mixture of stochastic dual power utilities.
\end{abstract}

\section{Introduction}

Decision making under uncertainty is often viewed as an optimization problem under choice criterium, and the available observed data as the result of the decision process and its evolution over the time. Most theories focus on the derivation of the "optimal decision" and its outcomes, but poor information

*With the financial support of the "Chaire Risque Financier" of the "Fondation du Risque" and the "Labex MME-DII"

${ }^{\dagger}$ Key Words. inverse thinking, the utility recovery problem, revealed preference/utility, characteristic process, consistent utility, progressive utility, portfolio optimization, duality, economic equilibrium, aggregated utility.

${ }^{\ddagger}$ Laboratoire de Probabilités, Statistique et Modélisation (LPSM, UMR CNRS 8001), Sorbonne Université

${ }^{\S}$ Laboratoire Analyse, Géomètrie et Applications (LAGA, UMR CNRS 7539), Université Paris 13 
is available on the preference criterium generating (yielding to) these observed data. The problem to recover the criterium from the data may be viewed as an inverse problem. Similar question was addressed by the economist Samuelson in the 40's [Sam38, Sam48], with the theory of "revealed preference" where the observable is the consumption process. Since then, the theory has been growing in interest under the pressure of the economic reality, which created news incentives for different approaches, see Chambers \& Echenique [CE16]. An example is the evolutionary economics by Arthur Art99]. Economies are considered as complex evolutionary systems, where the agents try to predict the outcomes of their actions, and how the market would be modified by their decisions.

This forward-looking viewpoint has been considered in many optimization problems outside of economics and finance, in the last few decades as a consequence of the expansion of computer science. The forward modelling allows anticipations on the future values of observables. But, the agents also need to adjust their (random) preferences over time, following an "inverse thinking" approach as has been suggested by Gomez-Ramirez [GR13]. The robustness of the method is obtained from a family of forward models consistent with the data. Problems generated by the e-commerce, see William \& al [WTKD04], belong to this family. The main problem is to build the utility function of a user from his search history and purchases on the Internet. This utility will then be used to target the user by proposing products or baskets of goods that we know are likely to be of interest to him and at specific prices evaluated from this utility function. As well, reasoning with preferences has been recognized as a particularly promising research direction for artificial intelligence, see Nielsen and Jensen [NJ04] or Qi, Xu, and Lafferty QXL14, and more generally in maching learning, see Friedman and Sandow [FH11], Chajewska, Koller, and Ormoneit CKO01 and Stahl Sta04 and Hibbard Hib12.

Our approach is therefore a learning approach, based on the observation of the outcomes of a "player" from several initial conditions, at many dates in the future; the question is what can be deduced about his utility at any time and what about time-concistency? The answer suggests to work in a general probabilistic framework which includes discrete frame, or semimartingale's frame (with jumps). Thus the results could apply also to the "Utility learning theory, Preference learning theory, Algorithmic Decision theory" much considered nowadays.

Dynamic utility was first considered by Musiela and Zariphopoulou [MZ03, MZ10a, MZ10b] (under the name of forward utility) for the study of optimization problem in financial market, see also Henderson and Hobson [HH07], and El Karoui and Mrad [EKM13] and El Karoui \& al [EKHM18] for a model with consumption. In this paper, there is no financial market and a priori no optimization problem, nevertheless we show that much stronger assumptions are necessary to make an analogy with financial market.

Let us briefly present the different steps of the revealed dynamic utility problem. By definition, the observable is a so-called dynamic positive characteristic process $\left\{X_{t}(x)\right\}$ considered for different values of its initial condition $x_{0}(x)=x>0$, and assumed to be increasing in $x$ (to be coherent with the expected utility criterium). The goal is to recover, from a given initial utility function $u$, a stochastic dynamic utility $\mathbf{U}=\{U(t, z), z>0\}$, "revealed optimally" in the sense that at any (stopping) time, the preference for the observable process is in mean equal to its value at time $0, \mathbb{E}\left(U\left(t, X_{t}(x)\right)\right)=u(x)$ : from the probabilistic dynamic view point, on a given filtered probability space, the performance process "U(t, $\left.X_{t}(x)\right)$ is a martingale". Focusing on the concavity of the utility criterium $\mathbf{U}$, tools of convex analysis play a key role, especially the invertible decreasing marginal utility $U_{z}(t, z)$. Its allows to define the convex Fenchel-Legendre transform $\widetilde{U}(t, y)$ of $U(t, x)$, by $U(t, z)-z U_{z}(t, z)=\widetilde{U}\left(t, U_{z}(t, z)\right)$, but also to linearize the recovery problem, by using the one to one correspondence between the class of dynamic utilities $\mathbf{U}$ (revealed by $X$ ) and the adjoint processes $Y$ candidate to play the role of $U_{z}\left(t, X_{t}\right)$, more precisely $\left\{Y_{t}\left(u_{z}(x)\right)=U_{z}\left(t, X_{t}(x)\right)\right\}$. Since the characteristic process is invertible, the triplet $\left.\left\{\left(u, X_{t}(x), U(t, z)\right)\right\}\right)$ is in one to one correspondence with the triplet $\left\{\left(u, X_{t}(x), Y_{t}\left(u_{z}(x)\right)\right\}\right.$. The goal is then to identify 
the class of the adjoint processes $\left\{Y_{t}(y)\right\}$ in bijection with a revealed dynamic utility $\mathbf{U}$, such that $U\left(t, X_{t}(x)\right.$ is a martingale. A quasi-necessary condition on the triplet $\left\{\left(u, X_{t}(x), Y_{t}(y)\right)\right.$ is " $\left\{X_{t}(x) Y_{t}(y)\right\}$ is a supermartingale for any $x, y$ and $\left\{X_{t}(x) Y_{t}\left(u_{z}(x)\right)\right\}$ is a martingale for $\left.y=u_{z}(x)\right)$ "; this last condition guarantees that the conjugate dynamic process $\left\{\widetilde{U}\left(t, Y_{t}(y)\right)\right\}$ read along the adjoint process $\left\{Y_{t}(y)\right\}$, is a martingale.

The paper is organized as follows. Section 1 formalizes the previous ideas, and develop some consequences of the martingale formulation of the revealed problem. This framework is illustrated by simple but interesting examples, including the dilatation operator. In the second part of this section, the characteristic process $x \mapsto X_{t}(x)$ is assumed increasing with values in $[0, \infty)$ and its inverse process is denoted $\xi(t, z)$. We adopt an algebraic point of view based on the so-called adjoint process defined by $\left.\left\{Y_{t}^{X}\left(u_{z}(x)\right)=U_{z}\left(t, X_{t}(x)\right)\right\}\right)$. By monotonicity of $X_{t}(x)$, the problem of the computation of the marginal utility reduces to the use of a change of variable formula: $U_{z}(t, z)=Y_{t}\left(u_{z}(\xi(t, z))\right)$. For a given pair $(u, \mathcal{X})$ there is a one to one correspondance between a dynamic utility and an adjoint process. The problem becomes to characterize the adjoint processes generating a revealed utility.

Section 2 is dedicated to this new problem. The previous change of variable gives a way, via a Stieltjes integral, to study the martingale properties of $U\left(t, X_{t}(x)\right)-u(x)=\int_{0}^{x} Y_{t}\left(u_{z}(z)\right) d_{z} X_{t}(z)$. We establish a necessary and sufficient martingale condition on the processes $\left\{X_{t}(x)\right\}$ and $\left\{Y_{t}(y)\right\}$ for the solvability of the recovery problem, Propositions 2.1 and 2.2 .

As usual in convex analysis, it is natural to associate the conjugate utility in assuming that the admissible adjoint process, $\left\{Y_{t}^{x}(y)\right\}$ is characteristic for the conjugate utility, i.e. $\left\{\widetilde{U}\left(t, Y_{t}^{x}(y)\right\}\right.$ is martingale. Then $U$ is said to be bi-revealed, and by the master equation, $\left\{x_{t}(x) Y_{t}^{x}\left(u_{z}(x)\right)\right\}$ is a martingale. More interesting for the applications is the "quasi-necessary" and sufficient condition for the solvability of birevealed utility, expressed in terms of supermartingales (Theorem 2.2 of the product $\left\{X_{t}(x) Y_{t}(y)\right\}$ for any $(x, y)$ (strong-suborthogonal condition), with martingale constraint along the curve $y=u_{z}(x)\left(u_{z^{-}}\right.$ orthogonality). In Section 2, no regularity in time on the characteristic process or dynamic utility or adjoint processes is assumed. In return, monotonicity for the functions $\left(X_{t}(x), U_{z}(t, z), Y_{t}(y)\right)$ with respect to their initial condition is fundamental. To our knowledge, this monotonicity has never been often considered in literature. Under these last conditions, we deepen the idea of dilatation of Section 1.1 .4 by taking as processes $\Lambda_{t}$ the limit (assumed to exist) of $\left\{X_{t}(x) / x\right\}$ near 0 and as $H_{t}$ the limit of $\left\{Y_{t}(y) / y\right\}$ for $y$ going to $\infty$. Since $\left\{X_{t}(x) Y_{t}\left(u_{z}(x)\right)\right\}$ is a martingale going to $\left\{\Lambda_{t} H_{t}\right\}$ when $x \rightarrow 0$, its is natural to assume that the limit is also a martingale. This allows us to place ourselves in an intrinsic universe where in addition of the previous assumption, the characteristic process and its dual are simply supermartingales, see Section 2.1.5.

In the last part of this section, we consider the Itô semimartingale framework. The characteristic $X$ and its adjoint $Y$ are assumed to be regular Itô's random fields; their monotonicity is provided by the regularity of the random coefficients. Under the quasi-necessary and sufficient condition of strong suborthogonality (Theorem 2.2 between $X$ and $Y$, we fully characterize the dynamics of the bi-revealed utility and its dual transform by Itô-Ventzel's formula. They are solutions of two second order SPDEs of Hamilton, Bellman, Jacobi (HJB) type, see Theorem 2.4 and Proposition 2.4. Then, in Section 2.3.1. we associate an optimization problem whose the dynamic utility is the value function. The control processes belong to the family of semimartingales in the suborthogonal cone of $\left\{Y_{t}(y)\right\}$ for any $y$.

Section 3, contains complementary results. First, we precise the technical regularity condtions necessary to solve the previous SPSEs. Second, we give a numerical approximation of the revealed utility SPDEs, based on the construction of the solution from two SDEs..

Third, we complete the study of the "intrinsic universe", obtained from the one considered so far by a well-chosen stochastic dilation of the utility process. The advantage is that in this new universe all the transformed processes are supermartingales and the calculations are much simpler especially when we 
are interested in the particular framework where the characteristic process and its adjoint are strongly orthogonal solutions of a decoupled system of SDEs yielding, under additional assumption, to a framework similar to financial market developed by the authors in [EKM13.

In Section 4, we revisit and solve completely the dynamic equilibrium problem as in He and Leland HL93, by considering it as a revealed utility problem where $Y$ is coupled with $X$ so that the monotonicity of $Y_{t}\left(z, u_{z}(z)\right)$ is lost. Nevertheless, under the general condition that is the characteristic and its adjoint are only strong suborthogonal, we prove that even if the environment is random, the dual problem at equilibrium is very easy to characterize: the adjoint process is linear in $y$ (geometric Brownian motion (GBM) in the Markovian case), Proposition 4.1 and the conjugate utilities are deterministic mixtures of stochastic dual power utilities, Theorem 4.3 , the primal utility is the value function of an optimal wealth allocation in sup-convolution Pareto problem, Theorem 4.4. Then, we discuss the consequences of this result.

\section{The forward revealed problem}

\subsection{Dynamic utilities, Observable processes and Time consistency.}

We start by reminding some definitions and properties of static and dynamic utility criterion, frequently used as performance measure in many areas, especially in economics and finance.

\subsubsection{Utility performances, and their observables}

Deterministic UTILITY FUnCTION The notion of utility function refers to a concave function $u$ on $\mathbb{R}^{+}$, positive, increasing, normalized by $u(0)=0$, whose range is $\mathbb{R}^{+}(u(+\infty)=\infty)$. An important role is played by its derivative $u_{z}$, also called marginal utility, assumed to be continuous, positive and decreasing on $] 0,+\infty[$, with range $] 0,+\infty[$, and satisfying Inada's conditions,

$$
u_{z}(+\infty)=0, \quad u_{z}(0)=+\infty
$$

As usual, the Fenchel-Legendre convex conjugate function $\tilde{u}(y)$ highlights some other aspects of the performance measure. The pair $(u, \tilde{u})$ satisfies the following system, where the first line is due to Legendre and the second line to Fenchel.

$$
\text { (Main equation) }\left\{\begin{array}{l}
\tilde{u}(y)=\sup _{z>0}(u(z)-y z), \quad u(z)=\inf _{y>0}(\tilde{u}(y)+y z) . \\
u(z)-z u_{z}(z)=\tilde{u}\left(u_{z}(z)\right), \quad u\left(-\tilde{u}_{y}(y)\right)+y \tilde{u}_{y}(y)=\tilde{u}(y)
\end{array}\right.
$$

In particular, since $\tilde{u}(y)>0$, then $u(z)>z u_{z}(z)>0$ and $z u_{z}(z) \rightarrow u(0)=0$. The range of the decreasing function $\tilde{u}(y)$ is $] 0,+\infty\left[\right.$ since $\tilde{u}(y) \rightarrow \infty$ when $y \rightarrow 0\left(\right.$ since $\left.\sup _{z>0} u(z)=+\infty\right)$ and $\tilde{u}(y) \rightarrow 0$ when $y \rightarrow+\infty\left(u(0)=0\right.$ and $\left.z u_{z}(z)>0\right)$.

A typical example is the family of power utility $u^{(\alpha)}(z)=\frac{z^{1-\alpha}}{1-\alpha}$ for $\alpha \in\left[0,1\left[\right.\right.$ and $u(z)=(\ln z)^{+}$for $\alpha=1$, whose relative risk aversion is the constant $\alpha$. Its Fenchel conjugate is the power function $\tilde{u}^{(\beta)}(y)=y^{(1-\beta)} /(\beta-1), \beta=1 / \alpha>1$, whose risk tolerance is the constant $\beta$.

DyNAmic UtiLities A dynamic utility should represent, possibly changing over time, individual preferences of an "agent" starting with a today's specification of his utility, $U(0, z)=u(z)$.

The preferences are affected over time by the available information represented by the filtration $\left(\mathcal{F}_{t}\right)_{t \geq 0}$ defined on the probability space $(\Omega, \mathbb{P}, \mathcal{F})$. The filtered probability space $\left(\Omega, \mathbb{P},\left(\mathcal{F}_{t}\right)\right)$ is assumed to satisfy usual conditions of right continuity and completeness. The filtration $\mathcal{F}_{0}$ is not necessarily assumed to be 
trivial. The $\sigma$-fields $\mathcal{O}$ of optional processes or $\mathcal{P}$ of predictable processes are generated by the families of adapted, respectively right-continuous or left-continuous processes.

A dynamic utility $\mathbf{U}$ is a collection of random utility functions $\{U(t, \omega, z)\}$ whose temporal evolution is "updated" in accordance with the new information $\left(\mathcal{F}_{t}\right)$, from an initial utility function $u(z)=U(0, z)$ eventually random if $\mathcal{F}_{0}$ is not trivial.

Definition 1.1. A dynamic utility $\mathbf{U}$ is a family of optional processes $\left\{U(t, z), z \in \mathbb{R}^{+}\right\}$(also called optional random field) such that $\mathbb{P}$.a.s., for every $t \geq 0$, the function $(z \rightarrow U(t, z))$ is a standard utility function with $U(t, 0)=0$.

- Its marginal utility $\mathbf{U}_{z}$ is the decreasing optional random field $\left\{U_{z}(t, z)\right\}$ with range $[0, \infty]$.

- Its conjugate utility $\widetilde{\mathbf{U}}$ is the convex optional random field, $\widetilde{U}(t, y)=\sup _{z>0}(U(t, z)-y z)$, (Master Equation) $\quad U(t, z)-z U_{z}(t, z)=\widetilde{U}\left(t, U_{z}(t, z)\right)$ and $\widetilde{U}_{y}\left(t, U_{z}(t, z)\right)=-z . \mathbb{P}$. a.s.

Characteristic process and adjoint process. This paper addresses the question of the recoverability of a dynamic utility from an observed process. Let us make two remarks at this stage:

- to recover a family of "utility functions" depending on a parameter $z$, at any time in the future, the "observable" process must also depend on a parameter through its initial conditions $x$. Such family will be called characteristic and denoted by the calligraphic symbols $\left\{X_{t}(x)\right\}$, for $(t, x) \in \mathbb{R}^{+} \times \mathbb{R}^{+}$.

- the pairing between concave utility and its Fenchel conjugate leads to match the characteristic process with "an adjoint process" helping to define the conjugate utility via the master equation (1.1).

Definition 1.2. - The characteristic process $\left\{X_{t}(x)\right\}$ is a positive optional process, parametrized by its initial condition $x \in \mathbb{R}^{+}$. By assumption $x \rightarrow X_{t}(x)$ is continuous in $x$, with boundary conditions $x_{t}(0)=0$, and $x_{t}(\infty)=\infty$.

- An adjoint process is an optional process, continuous in $y$, with range $[0, \infty)$. In particular, $Y_{t}(0)=0$, and $Y_{t}(\infty)=\infty$.

In general the asymptotic limits concern 0 for the characteristic process and the infinite for the adjoint process.

There is nevertheless a difference between the two processes, since a priori the characteristic process is given while the adjoint is to be chosen.

\subsubsection{Time-stability for revealed utilities and martingales}

The first step is to define the relationship between the characteristic process and the dynamic utility, $\left\{U(t, z),(t, z) \in \mathbb{R}^{+} \times \mathbb{R}^{+}\right\}$to be recovered. The dynamic point of view requires a time-stability on the "expected utility" (performance) of the characteristic process in the future. Time-stability is not a new principle, since it is already found in the seminal paper of L.Bachelier in 1900 Bac00, as a natural assumption on the mechanism of prices formation, and of course in mathematical finance in relation with the no-arbitrage assumption. This property is also the cornerstone of stochastic control problem, without reference to economics or finance, as well as the use of concave criterium, ([EKPY12]). This principle is known as dynamic programming principle, or Bellman principle.

Mathematically, this means that for any bounded stopping time $\tau$ (such that the positive random variable $U\left(\tau, X_{\tau}(x)\right)$ is integrable), $\mathbb{E}\left[U\left(\tau, X_{\tau}(x)\right)\right]=u(x)$ and then, for any bounded stopping times, $\sigma \leq \tau$, $\mathbb{E}\left[U\left(\tau, X_{\tau}(x)\right)\right]=\mathbb{E}\left[U\left(\sigma, X_{\sigma}(x)\right)\right]$. 
Martingale properties for revealed utilities A more flexible formulation is given in a pathwise point of view: "the process $\left\{U\left(t, X_{t}(x)\right)\right\}$ is a (strong) martingale". By means of the monotonic change of variable $x \rightarrow X_{t}(x)$, we make the "curve" $\left\{U\left(t, X_{t}(x)\right)\right\}$ constant in expectation, hence the name of characteristic process for $\left\{X_{t}(x)\right\}$.

Martingale properties are taken as central in the definition of revealed dynamic utility. The assumptions are reinforced by the martingale properties of the conjugate utility along of the adjoint process. Note that the existence (and of course the uniqueness) of such utilities is not a priori guaranteed, as explained in Subsection 2.2 .

Definition 1.3 (Revealed dynamic utility). Let $\left\{X_{t}(x)\right\}$ be a characteristic process and $u$ a given utility at time 0. (i) A dynamic utility $\{U(t, z)\}$ is said to be revealed by $(u, X)$ if:

$$
\forall x \in(0, \infty), \quad\left\{U\left(t, X_{t}(x)\right)\right\} \text { is a positive martingale. }
$$

(ii) More generally, a dynamic utility $\{U(t, z)\}$ is said to be bi-revealed by $(u, X)$ if:

$$
\forall x \in(0, \infty), \quad\left\{U\left(t, X_{t}(x)\right)\right\} \text { and }\left\{X_{t}(x) U_{z}\left(t, X_{t}(x)\right)\right\} \text { are positive martingales. }
$$

(iii) The process $Y_{t}^{X}(y)=U_{z}\left(t, X_{t}\left(-\tilde{u}_{y}(y)\right)\right)$ is the so-called $X$-adjoint process.

Then, $\{U(t, z)\}$ is bi-revealed iff $\{U(t, z)\}$ is revealed by $(u, X)$, and $\{\widetilde{U}(t, y)\}$ is revealed by $\left(\tilde{u}, Y^{X}\right)$.

The last assertion is a simple consequence of the Master equation (1.3.

\subsubsection{Examples of revealed utilities}

ELEMENTARY EXAMPLES The following elementary examples of characteristic (observed) processes are used to give a first insight of the problem of revealed utility. The case of constant process, corresponding to "to do nothing in finance", is very illustrative. The case of linear characteristic processes yields to a very simple characterization, that is why it is also used in economics and finance. Similar results can be easily extended to convex increasing process.

Proposition 1.1. (i) Assume the characteristic process to be constant in time: $X_{t}(z)=z, \forall t$.

A dynamic utility $\{U(t, z)\}$ is a revealed utility, if and only if the marginal utility $\left\{U_{z}(t, z), z>0\right\}$ is a martingale. Then, $\{U(t, z)\}$ is a bi-revealed utility.

(ii) Assume $X$ to be linear in $x:\left\{X_{t}(x)=x X_{t}\right\}$. Then, $\mathbf{U}$ is a revealed utility, if and only if $\mathbf{U}$ is a bi-revealed utility; equivalently, there exists an adjoint process $\left\{Y_{t}(y)\right\}$ such that for any $y,\left\{X_{t} Y_{t}(y)\right\}$ is a martingale. Then, $\left\{Y_{t}(y)\right\}$ is a characteristic process for the conjugate utility process $\widetilde{\mathbf{U}}$ and $Y_{t}\left(u_{z}(x)\right)=$ $U_{z}\left(t, x X_{t}\right), \forall x$.

(iii) Assume $\left\{X_{t}(x)\right\}$ to be concave, increasing and differentiable in $x$ with differential $\left\{X_{x}(t, x)\right\}$. Then, $\left\{U^{X}(t, z)=U\left(t, X_{t}(z)\right)\right\}$ is revealed by $(u, z)$ if and only if $\left\{U_{z}\left(t, X_{t}(z)\right) X_{x}(t, z)=Y_{t}\left(u_{z}(z)\right) X_{x}(t, z)\right\}$ is a martingale; and then $\left\{U^{x}(t, z)\right\}$ is also bi-revealed. But $\mathbf{U}$ is only a revealed utility, and is bi-revealed iff in addition $\left\{Y_{t}\left(u_{z}(z)\right) X_{t}(z)\right\}$ is a martingale.

Proof. (i) Assume the utility process $\{U(t, x)\}$ to be a martingale. By the Master equation $1.3,0 \leq$ $z U_{z}(t, z) \leq U(t, z) \leq U\left(t, z_{\max }\right), z \in\left[0, z_{\max }\right]$. By Lebesgue's derivative theorem, the martingale property can be extended to the derivative random field $\left\{U_{z}(t, z)\right\}$ and then to $\left\{z U_{z}(t, z)\right\}$. Conversely, if the $z$ decreasing process $\left\{U_{z}(t, z)\right\}$ is a martingale, by Fubini's Theorem $\left\{U(t, x)-U\left(t, x_{0}\right)\right\}$ is also a martingale, with expectation $u(x)-u\left(x_{0}\right)$. Thanks to the monotonicity of utility functions, $U\left(t, x_{0}\right)$ decreases to 0 when $x_{0}$ goes to 0 , and the martingale property remains valid at the limit for $\{U(t, x)\}$. By the Master equation (1.3), $\mathbf{U}$ is bi-revealed from $(u, z)$. Then, by Definition 1.3 , $\mathbf{U}$ is bi-revealed by $(u, z)$.

(ii) If $X_{t}(x)=x X_{t}$, the change of variable (also called change of numeraire in finance) $x \rightarrow x / X_{t}$ yields to 
a new dynamic utility, $U^{X}(t, z)=U\left(t, z X_{t}\right)$ which is a martingale when $U$ is revealed, with characteristic process $X_{t}(x) / X_{t}=x$. By (i) $U^{X}(t, z)$ is a martingale if and only if $U_{z}^{X}(t, x)=X_{t} U_{z}\left(t, x X_{t}\right)=$ $X_{t} Y_{t}\left(u_{z}(x)\right)$ is a martingale. This condition is then sufficient for $\mathbf{U}$ being bi-revealed by $\left(u,\left\{x X_{t}\right\}\right)$.

(iii) When the characteristic process is concave, differentiable and increasing in $x$ and $\left(x \rightarrow X_{x}(t, x)\right)$ is decreasing in $x)$, the random field $z \rightarrow U\left(t, X_{t}(z)\right)$ is still concave. Then, $\left\{U^{X}(t, z)=U\left(t, X_{t}(z)\right)\right\}$ is a dynamic utility which is a martingale. From i), this is equivalent to the martingale property of its derivative $\left\{U_{z}\left(t, X_{t}(x)\right) X_{x}(t, z)=Y_{t}\left(u_{z}(z)\right) X_{x}(t, z)\right\}$. Then, the dynamic utility $\mathbf{U}^{X}$ is bi-revealed by $(u, z)$. But the dynamic utility $\mathbf{U}$ itself is only revealed by $(u, X)$, and bi-revealed if and only if in addition the process $\left\{Y_{t}\left(u_{z}(z)\right) X_{t}(z)\right\}$ is a martingale.

\subsubsection{Dilatation of revealed and bi-revealed utility}

A way to generate new revealed utilities, from a given dynamic utility and its characteristic process considered as the root of the transformation, is to use the dilation operator, well-known in convex analysis, because it simply acts in a linear manner on the Fenchel-conjugate utility.

The dilation operator is defined on real utility functions, for any real parameter $\lambda>0$ by $u^{\lambda}(z)=$ $\frac{1}{\lambda} u(z \lambda), \quad u_{z}^{\lambda}(z)=u(z \lambda), \quad \tilde{u}^{\lambda}(y)=\frac{1}{\lambda} \tilde{u}(y)$.

In dynamic utility framework, the dilation factor is an adapted positive process $\left\{\Lambda_{t}\right\}$, expected to transform a revealed utility with given characteristic process into a revealed utility with a modified characteristic process. This transformation has been successfully used in the study of convex risk measures by P. Barrieu and N. El Karoui, BEK05]. In finance, this transformation is relative to the technics of "change of numeraire, and change of probability measure", H. Geman \& al GEKR95.

Dilatation of revealed utilities Let $\{U(t, z)\}$ be a dynamic utility, and $\left\{\Lambda_{t}\right\}$ a dilatation factor. The stochastic version of the dilated utility is $\left\{U^{\Lambda}(t, z)=\Lambda_{t}^{-1} U\left(t, z \Lambda_{t}\right)\right\}$ whose marginal utility is $\left\{U_{z}^{\Lambda}(t, z)=\right.$ $\left.U_{z}\left(t, z \Lambda_{t}\right)\right\}$ and the Fenchel conjugate utility is $\left\{\widetilde{U}^{\Lambda}(t, y)=\Lambda_{t}^{-1} \widetilde{U}(t, y)\right\}$.

When $\{U(t, z)\}$ is a revealed utility with characteristic process $\left\{X_{t}(x)\right\}$ and adjoint process $\left\{Y_{t}\left(u_{z}(x)\right)=\right.$ $\left.U_{z}\left(t, X_{t}(x)\right)\right\}$, the question is to if the martingale property of $\left\{U\left(t, X_{t}(x)\right)\right\}$ is propagated by dilatation". A candidate to be a $\Lambda$-characteristic process is $\left\{X_{t}^{\Lambda}(x)=\Lambda_{t}^{-1} X_{t}(x)\right\}$, since $U^{\Lambda}\left(t, X_{t}^{\Lambda}(x)\right)=\Lambda_{t}^{-1} U\left(t, X_{t}(x)\right)$. If $\left\{\Lambda_{t}\right\}$ is a $\mathbb{P}$-martingale, then $\left\{\Lambda_{t} U^{\Lambda}\left(t, X_{t}^{\Lambda}(x)\right)=U\left(t, X_{t}(x)\right)\right\}$ is a $\mathbb{P}$-martingale and $\left\{U^{\Lambda}\left(t, X_{t}^{\Lambda}(x)\right)\right\}$ is a martingale with respect to the new probability measure $\mathbb{Q}=\Lambda . \mathbb{P}$. Then, the pair $\left(U^{\Lambda}, X^{\Lambda}\right)$ is $\mathbb{Q}^{\Lambda}$ revealed. If $\left\{\Lambda_{t}\right\}$ is not a $\mathbb{P}$-martingale, we can replace $L_{t}=\Lambda_{t}$ by a martingale $\left\{L_{t}=\Lambda_{t} H_{t}\right\}$, and introduce a new dilated dynamic utility $\left\{U^{\Lambda, H}(t, z)=H_{t}^{-1} U^{\Lambda}(t, z)=L_{t}^{-1} U\left(t, z \Lambda_{t}\right)\right\}$. Since the new dynamic utility is $\left\{H_{t}^{-1} U^{\Lambda}(t, z)\right\}$, all the required martingale properties are satisfied under the probability measure $d \mathbb{Q}^{\Lambda, H}=H_{t} \Lambda_{t} / H_{0} \Lambda_{0} d \mathbb{P}$ on $\mathcal{F}_{t}, \forall t$..

Proposition 1.2. Let $(u, X, U)$ be a revealed utility, and $\left\{\left(H_{t}, \Lambda_{t}\right)\right\}$ a pair of adapted processes such that le product $\left\{H_{t} \cdot \Lambda_{t}\right\}$ is a $\mathbb{P}$-martingale. Put $L_{t}=H_{t} \cdot \Lambda_{t} / H_{0} \cdot \Lambda_{0}$ and define a new probability measure by $d \mathbb{Q}^{\Lambda, H}=L_{t} d \mathbb{P}$ on $\mathcal{F}_{t}$ for any $t$.

(i) Then the triplet $\left(u, X^{\Lambda}, U^{\Lambda, H}\right)$ is a $\mathbb{Q}^{\Lambda, H}$-revealed utility iff

$$
U^{\Lambda, H}(t, z)=\left(H_{t} \Lambda_{t}\right)^{-1} U\left(t, z \Lambda_{t}\right), \quad X_{t}^{\Lambda}(x)=\Lambda_{t}^{-1} X_{t}(x), \quad Y_{t}^{H}(y)=H_{t}^{-1} Y_{t}(y), \forall x, y, z, t>0 .
$$

(ii) In addition, since $\widetilde{U}^{\Lambda, H}(t, y)=\left(H_{t} \Lambda_{t}\right)^{-1} \widetilde{U}\left(t, y H_{t}\right), \widetilde{U}^{\Lambda, H}\left(t, Y_{t}^{H}(y)\right)$ is a $\mathbb{Q}^{\Lambda, H}$ martingale, if and only if $\widetilde{U}\left(t, Y_{t}(y)\right.$ is a $\mathbb{P}$-martingale.

In this proposition, the family of processes $(\Lambda, H)$ is quite general, but if $\left\{X_{t}(x) \sim \Lambda_{t} x\right\}$ near to 0 , and $\left\{Y_{t}(y) \sim H_{t} y\right\}$ (with $\Lambda_{t} H_{t}$ martingale) the dilated problem is considerably simplified see Theorem 2.3 . 


\subsection{Monotonicity, Adjoint process and Pathwise marginal utility}

The previous examples have in common the monotonicity of the characteristic process in $x$, and of the adjoint process defined by $\left\{Y_{t}^{X}\left(u_{z}(x)\right)=U_{z}\left(t, X_{t}(x)\right)\right\}$. The role of this property is not surprising, since the monotonicity and the concavity of $z \rightarrow U(t, z)$ cannot be obtained only from the martingale property of $\left\{U\left(t, X_{t}(x)\right)\right\}$, even if the initial condition $u$ is concave and increasing. This difficulty comes from the forward point of view for which comparison results are difficult to obtain in general.

\subsubsection{Monotonicity and Algebraic Compatibility}

The characteristic process $\left\{X_{t}(x)\right\}$ is assumed to be an optional random field, increasing in $x$ with range $[0, \infty)$. Then, a.s. the function $x \rightarrow X_{t}(x)$ is continuous and invertible, with optional increasing inverse flow $\xi(t, z)$ such that $X_{t}(\xi(t, z))=z$. Then, the same property of monotonicity is satisfied by the so-called adjoint process $\left\{Y_{t}^{x}(y)\right\}$, defined by $Y_{t}^{x}\left(u_{z}(x)\right)=U_{z}\left(t, X_{t}(x)\right)$. Observe that necessarily $Y_{t}^{x}\left(u_{z}(\xi(t, z))(=\right.$ $\left.U_{z}(t, z)\right)$ has to be integrable in a neighborhood of $z=0$ as any marginal utility function.

More generally, we are interested in processes defined without reference to any dynamic utility U. So, we concentrate our attention on the adjoint process $Y_{t}^{x}\left(u_{z}(x)\right)$ and try to recover the marginal utility by pathwise algebraic formula.

Definition 1.4 (Algebraic Compatibility). (i) The class $\mathcal{A}$ is the family of admissible triplet $(u, X, Y)$, where $u$ is a utility function, the processes $x \mapsto X_{t}(x), y \mapsto Y_{t}(y)$ are increasing with range $[0, \infty)$, with integrability in 0 of $Y_{t}\left(u_{z}(\xi(t, z))\right.$.

(ii) An admissible triplet $(u, X, Y) \in \mathcal{A}$ defines a dynamic utility $\{U(t, z)\}$, through the "first order condition":

$$
U_{z}(t, z)=Y_{t}\left(u_{z}(\xi(t, z)) \text { so that } U\left(t, x_{t}(x)\right)=\int_{0}^{x} Y_{t}\left(u_{z}(z)\right) d_{z} X_{t}(z), \quad U(t, 0)=0 .\right.
$$

(iii) Let $\zeta(t, y)$ be the inverse flow of $y \mapsto Y_{t}(y)$. By algebraic duality, the Fenchel conjugate is given by:

$$
\tilde{U}_{y}(t, y)=-x_{t}\left(-\tilde{u}_{y}(\zeta(t, y)), \quad \widetilde{U}\left(t, Y_{t}(y)\right)=\int_{y}^{\infty} x_{t}\left(-\tilde{u}_{y}(z)\right) d_{z} Y_{t}(z), \quad \lim _{y \rightarrow \infty} \tilde{U}(t, y)=0 .\right.
$$

Such dynamic utility is said to be $(u, X, Y)$-compatible. The conjugate is also $(\tilde{u}, Y, X)$-compatible.

Surprisingly, in economics and finance the monotonicity property is rarely highlighted, although it is satisfied by almost all known solutions.

\subsubsection{Forward starting dates and time consistency}

As usual in the dynamic viewpoint, it is appropriate to consider different starting dates, and different starting point in the future. Then, a time consistency is required between these different processes. This is a general principle that we find in many situations, in the theory of stochastic differential equation with respect of their initial condition, in the study of Markov processes, or in optimization problems where it is known as the dynamic programming principle.

This forward extension requires to define a family $X_{t}(x, s)$ of processes, starting from $x$ at time $s$, satisfying the time consistency, or semi-group property, that for $t \geq s, X_{t}(x)=X_{t}\left(X_{s}(x), s\right)$. The monotonicity assumption plays a key role, in the definition of the adapted inverse process $\{\xi(t, z)\}$ of $\left\{X_{t}(x)\right\}$ and the inverse process $\left\{\widetilde{\xi}(t, \zeta)=\left(Y_{\text {. }}\right)^{-1}(t, \zeta)\right\}$ of $\left\{Y_{t}(y)\right\}$. This semi-group relation imposes that $X_{t}(x, s)=$ $\chi_{t}(\xi(s, x))$. Similarly, $Y_{t}(y, s)=Y_{t}(\widetilde{\xi}(s, y))$. 
Starting with an admissible triplet $(u, X, Y) \in \mathcal{A}$ generating a dynamic utility $\mathbf{U}$, the problem is to deduce a new triplet starting with initial condition $(s, z)$, coherent with this data, in particular with the same associated dynamic utility $U(t, z)$. Then, we justify also briefly that the revealed property is maintained.

Proposition 1.3 (Forward and Backward Formulation). Let $\mathbf{U}$ be a dynamic utility compatible with $(u, X, Y) \in \mathcal{A}$. (i) The new forward characteristic processes, starting from $x$ at the date $s$ are defined by for any $t \geq s$ by

$$
X_{t}(x, s):=X_{t}(\xi(s, x)), \quad Y_{t}(y, s):=Y_{t}(\widetilde{\xi}(s, y)),
$$

with the semi-group property, $X_{t}(x)=X_{t}\left(X_{s}(x), s\right), \quad Y_{t}(y)=Y_{t}\left(Y_{s}(y), s\right)$.

(ii) The system $\left(U(s, z), X_{t}(x, s), Y_{t}(y, s), t \geq s\right)$ is compatible with the stochastic utility $U(t, z, s)=$ $U(t, z)$.

(iii) Moreover, if $\mathbf{U}$ is revealed by $\left(u, X_{t}(x), Y_{t}(y)\right)$, then for any date $s$, and starting point $x$, for $t \geq s$, $U$ is still a revealed utility, starting from $\left(U(s, x), X_{t}(x, s), Y_{t}(x, s)\right)$.

Proof. (i) -(ii) The verification of the compatibility of $U(t, z)$ with the new processes relies on the verification of the algebraic identity $U_{z}\left(t, X_{t}(s, x)\right)=Y_{t}\left(U_{z}(s, x), s\right)$; by the semi-group property, this equality becomes $U_{z}\left(t, X_{t}(x)\right)=Y_{t}\left(U_{z}\left(X_{s}(x), s\right)\right)=Y_{t}\left(Y_{s}\left(u_{z}(z), s\right)=Y_{t}\left(u_{z}(z)\right)\right.$, which still holds thanks to the algebraic compatibility.

(iii) The algebraic point of view needs to be completed by the revealed point of view, introduced in the beginning of this subsection as a time stability in expectation of the performance of the characteristic process. Mathematically, this means that for bounded stopping times $\tau \geq \sigma$ (such that the positive random variable $U\left(\tau, X_{\tau}(x)\right)$ is integrable $), \mathbb{E}\left[U\left(\tau, X_{\tau}(x)\right)\right]=\mathbb{E}\left[U\left(\sigma, X_{\sigma}(x)\right)\right]=u(x)$.

Suppose now that $\sigma=s$ is viewed as a starting date and that $x$ is replaced by a $\mathcal{F}_{\sigma}$-bounded random variable $\eta_{\sigma}$, with only two values $\eta_{\sigma}=x_{1} \mathbf{1}_{A_{1}}+x_{2} \mathbf{1}_{A_{2}}$, with $\mathbf{1}_{A_{1}}+\mathbf{1}_{A_{2}}=1$ and $\left(A_{1}, A_{2}\right) \in \mathcal{F}_{\sigma}$. Then, $X_{\tau}\left(\eta_{s}\right)=X_{\tau}\left(x_{1}\right) \mathbf{1}_{A_{1}}+X_{\tau}\left(x_{2}\right) \mathbf{1}_{A_{2}}$ and $U\left(\tau, X_{\tau}\left(\eta_{s}\right)\right)=\mathbf{1}_{A_{1}} U\left(\tau, X_{\tau}\left(x_{1}\right)\right)+\mathbf{1}_{A_{2}} U\left(\tau, X_{\tau}\left(x_{2}\right)\right)$. So we still have that $E\left[U\left(\tau, X_{\tau}\left(\eta_{s}\right)\right)\right]=\mathbb{E}\left[U\left(s, \eta_{s}\right)\right]$. By continuity in $x$, this property can be extended to any $\mathcal{F}_{\sigma^{-}}$ measurable random variable. Reading this property differently shows that the dynamic utility starting from $\left(s, \eta_{s}\right)$ is a revealed utility.

\section{Solvability of the recovery problem}

Let $\mathbf{U}$ be the compatible dynamic utility defined by $(u, X, Y) \in \mathcal{A}$. The main issue of this section is to give conditions on $(u, X, Y)$ under which $\mathbf{U}$ is a revealed (resp. bi-revealed) utility, that is $\left\{U\left(t, X_{t}(x)\right\}\right.$ is a martingale, as well as $\left\{X_{t}(x) U_{z}\left(t, X_{t}(x)\right\}\right.$ in the bi-revealed case.

\subsection{Conditions for the solvability of the recovery problem}

We have seen that for a convex differentiable characteristic process $\left\{X_{t}(x)\right\}$ with derivative $\left\{X_{x}(t, x)\right\}$ $\left(X_{x}(t, 0)=1\right)$, the martingale property of $\left\{X_{x}(t, x) Y_{t}\left(u_{z}(x)\right)\right\}$ is a necessary and sufficient condition for solving the recovery problem. In fact the sufficient condition does not use the convexity of $\left\{X_{t}(x)\right\}$, and still holds also for only differentiable characteristic process. When the characteristic process is not differentiable, we have to rewrite the "first order condition" in a more general formulation.

\subsubsection{Solvability conditions for recovery problem}

In the general case, we use the rate of variation in place of the derivative, and the theorem of intermediate values. For a $(u, X, Y)$-compatible dynamic utility $\mathbf{U}$, it is easy to control the $x$-variation of $U\left(t, X_{t}(x)\right)$ with the help of the process $Y$. 
Lemma 2.1. There exists an optional process $\left\{\psi_{t}\left(x, x^{\prime}\right)\right\}$ such that for any $\left(x<x^{\prime}\right)$

$$
U\left(t, x_{t}\left(x^{\prime}\right)\right)-U\left(t, x_{t}(x)\right)=\left(x_{t}\left(x^{\prime}\right)-X_{t}(x)\right) Y_{t}\left(u_{z}\left(\psi_{t}\left(x, x^{\prime}\right)\right)\right)
$$

Proof. The proof is a simple consequence of the theorem of the intermediate values,

$$
\left\{\begin{array}{l}
U\left(t, z^{\prime}\right)-U(t, z)=\left(z^{\prime}-z\right) U_{z}\left(t, \psi_{t}\left(z, z^{\prime}\right)\right), \quad \psi_{t}\left(z, z^{\prime}\right) \in\left(z, z^{\prime}\right), z^{\prime}>z>0 \\
\psi_{t}\left(z, z^{\prime}\right)=\left(U_{z}\right)^{-1}\left(t, \Delta U\left(t,\left(z, z^{\prime}\right)\right)\right), \quad \Delta U\left(t,\left(z, z^{\prime}\right)\right)=\frac{U\left(t, z^{\prime}\right)-U(t, z)}{z^{\prime}-z}
\end{array}\right.
$$

Then, with $x<x^{\prime}$, and $\left(z=X_{t}(x), z^{\prime}=X_{t}\left(x^{\prime}\right)\right)$, there exists an optional process $\xi_{t}\left(z, z^{\prime}\right) \in\left[X_{t}(x), X_{t}\left(x^{\prime}\right)\right]$ such that, $U\left(t, X_{t}\left(x^{\prime}\right)\right)-U\left(t, X_{t}(x)\right)=\left(X_{t}\left(x^{\prime}\right)-X_{t}(x)\right) U_{z}\left(t, \xi_{t}\left(z, z^{\prime}\right)\right)$

By a change of variable, $\xi_{t}\left(z, z^{\prime}\right)$ can be sent into $\psi_{t}\left(x, x^{\prime}\right)$ in the interval $\left(x, x^{\prime}\right)$ by the formula $\xi_{t}\left(z, z^{\prime}\right)=$ $x_{t}\left(\psi_{t}\left(x, x^{\prime}\right)\right)$. Thus, $U_{z}\left(t, \xi_{t}\left(z, z^{\prime}\right)\right)=U_{z}\left(t, X_{t}\left(\psi_{t}\left(x, x^{\prime}\right)\right)\right)=Y_{t}\left(u_{z}\left(\psi_{t}\left(x, x^{\prime}\right)\right)\right)$.

Then, for any revealed utility, the left hand side is a martingale and so, by equation (2.1) for $x^{\prime}>x$, the right hand side " $\left(X_{t}\left(x^{\prime}\right)-X_{t}(x)\right) Y_{t}\left(u_{z}\left(\psi_{t}\left(x, x^{\prime}\right)\right)\right) "$ is a martingale. Our main result is that this condition is also sufficient.

AbStRaCt RESUlt: The argument uses the approximation of the Stieltjes integral $\int_{x_{0}}^{x} Y_{t}\left(u_{z}(z)\right) d_{z} x_{t}(z)$ defined on a compact interval $\left[x_{0}, x\right]$ with the help of Darboux sums obtained as follows: we start with a partition of the interval $\left[x_{0}, x\right]$ into $N$ subintervals $\left.] z_{n}, z_{n+1}\right]$ where the mesh approaches zero, and we consider the following sequences

$$
\bar{S}_{t}^{N}\left(x_{0}, x\right)=\sum_{n=0}^{N-1} Y_{t}\left(u_{z}\left(\bar{z}_{t}^{n}\right)\right)\left(X_{t}\left(z_{n+1}\right)-X_{t}\left(z_{n}\right)\right),
$$

where $\bar{z}_{t}^{n}$ is a random variable in the interval $\left[z_{n}, z_{n+1}\right]$. Given the continuity of $z \rightarrow Y_{t}\left(u_{z}(z)\right)$ and $z \rightarrow X_{t}(z)$, Darboux's Theorem states that all these sums converge to the Stieltjes integral when the mesh goes to 0 .

Proposition 2.1. Let $(u, X, Y) \in \mathcal{A}$ be an admissible triplet generating the dynamic utility $\mathbf{U}$.

$\mathbf{U}$ is a revealed utility if and only if for any $\left(x<x^{\prime}\right)$, there exists an optional process $\left\{\psi_{t}\left(x, x^{\prime}\right)\right\}$ with $x \leq \psi_{t}\left(x, x^{\prime}\right) \leq x^{\prime}$, such that for:

the process $\left\{\left(X_{t}\left(x^{\prime}\right)-X_{t}(x)\right) Y_{t}\left(u_{z}\left(\psi_{t}\left(x, x^{\prime}\right)\right)\right)\right\}$ is a martingale.

Proof. We use the approximations based on Darboux' sums centered around the processes $\bar{z}^{n}(t)=$ $\psi_{t}\left(z_{n}, z_{n+1}\right)$. By assumption, these Darboux approximations $S_{t}^{N}\left(x_{0}, x\right)$ are finite sum of positive martingales, and then also positive martingales. By the positive Fubini's Theorem, we can interchange limit and expectation so that the martingale property is preserved and $\int_{x_{0}}^{x} Y_{t}\left(u_{z}(z)\right) d_{z} x_{t}(z)$ is a martingale, with expectation $\int_{x_{0}}^{x} u_{z}(z) d z=u(x)-u\left(x_{0}\right)$.

Once again, by monotonicity, when $x_{0} \rightarrow 0$, the random variables $\int_{x_{0}}^{x} Y_{t}\left(u_{z}(z)\right) d_{z} x_{t}(z)$ go to a limit with finite expectation. So, the Stieltjes integral is well-defined up to 0 and $\left\{\Psi_{0}^{X}(t, x)=\int_{0}^{x} Y_{t}\left(u_{z}(z)\right) d_{z} x_{t}(z)\right\}$ is a martingale. The revealed utility process is then given by $U(t, z)=\int_{0}^{z} Y_{t}\left(u_{z}(\xi(t, x)) d x\right.$.

Similar arguments can be developed for the conjugate utility function.

\subsubsection{Efficient sufficient conditions and main result}

In the previous result, the existence of a process $\psi_{t}\left(z, z^{\prime}\right)$ satisfying the martingale property can be difficult to verify. The proof based on Darboux' approximations suggests to refer to the most common Darboux 
sums,

$$
\left\{\begin{array}{l}
S_{t}^{N, \text { up }}\left(x_{0}, x\right)=\sum_{n=0}^{N-1} Y_{t}\left(u_{z}\left(z_{n}\right)\right)\left(x_{t}\left(z_{n+1}\right)-x_{t}\left(z_{n}\right)\right), \\
S_{t}^{N, \text { down }}\left(x_{0}, x\right) \leq \bar{S}_{t}^{N}\left(x_{0}, x\right) \leq S_{t}^{N, u p}\left(x_{0}, x\right), \\
S_{t}^{N, \text { down }}\left(x_{0}, x\right)=\sum_{n=0}^{N-1} Y_{t}\left(u_{z}\left(z_{n+1}\right)\right)\left(X_{t}\left(z_{n+1}\right)-x_{t}\left(z_{n}\right)\right) .
\end{array}\right.
$$

The martingale assumptions are modified into only almost sufficient conditions that these processes are respectively supermartingales and submartingales. Since both sequences have the same limit, this limit is expected to be a martingale.

Proposition 2.2. Let $(u, X, Y) \in \mathcal{A}$ be a triplet generating the dynamic utility $\mathbf{U}$.

If for any $x^{\prime}>x$,

- the process $\left\{Y_{t}\left(u_{z}(x)\right)\left(X_{t}\left(x^{\prime}\right)-X_{t}(x)\right)\right\}$ is a supermartingale,

- the process $\left\{Y_{t}\left(u_{z}\left(x^{\prime}\right)\right)\left(X_{t}\left(x^{\prime}\right)-X_{t}(x)\right)\right\}$ is a submartingale,

then, the dynamic utility $\mathbf{U}$ is a revealed utility.

Proof. Let $0<x_{0}<x$ and consider a partition of the interval $\left[x_{0}, x\right]$ into $N$ subintervals $\left.] z_{n}, z_{n+1}\right]$ where the mesh approaches zero. We approach the integral $\int_{x_{0}}^{x} Y_{t}\left(u_{z}(z)\right) d_{z} x_{t}(z)$ by above, respectively by below, by the Darboux sums $S_{t}^{N, u p}\left(x_{0}, x\right)$, respectively by $S_{t}^{N, \text { down }}\left(x_{0}, x\right)$. Thanks to the monotonicity of the processes $\mathbf{Y}$ and $\left\{X_{t}(x)\right\}$, the Darboux sums $S_{t}^{N \text {,down }}\left(x_{0}, x\right)$ and $S_{t}^{N, u p}\left(x_{0}, x\right)$ are bounded above, and converge a.s. to the Stieltjes integral $\int_{x_{0}}^{x} Y_{t}\left(u_{z}(z)\right) d_{z} X_{t}(z)$.

Furthermore, by assumption, the $\operatorname{sum} S_{t}^{N, u p}\left(x_{0}, x\right)$ is a positive supermartingale, while the sum $S_{t}^{N, \text { down }}\left(x_{0}, x\right)$ is a positive submartingale. By the positive Fubini's theorem, for fixed $x_{0}>0$, one can interchange the $\lim _{N \rightarrow \infty}$ and the expectation to justify that the sub- and super- martingale properties are preserved at the limit. So, $\int_{x_{0}}^{x} Y_{t}\left(u_{z}(z)\right) d_{z} x_{t}(z)$ is a martingale, with expectation $\int_{x_{0}}^{x} u_{z}(z) d z=u(x)-u\left(x_{0}\right)$. Once again, by monotonicity, the integrals $\int_{x_{0}}^{x} Y_{t}\left(u_{z}(z)\right) d_{z} x_{t}(z)$ go to a limit with finite expectation. So, the Stieltjes integral is well-defined up to 0 and $\left\{\Psi^{x}(t, x)=\int_{0}^{x} Y_{t}\left(u_{z}(z)\right) d_{z} X_{t}(z)\right\}$ is a martingale; then the process $\left\{U(t, z)=\Psi^{x}(t, \xi(t, z))\right\}$ is a revealed dynamic utility.

\subsubsection{Orthogonality conditions: definitions and remarks}

In the characterization of revealed utility, we will often encounter properties such as "the product of two processes is a martingale or a supermartingale". This kind of assumption is frequently used for martingale processes and associated with a notion of orthogonality, due to the cancellation of their quadratic variation. ThE ROLE OF INITIAL CONDITIONS When the orthogonality concerns the product of two martingales $\left\{M_{t}, N_{t}\right\}$, it is not important to precise their initial conditions, because $\left(M_{t} \cdot N_{t}\right)$ is a martingale, if and only if $\left(\left(M_{t}-M_{0}\right) \cdot\left(N_{t}-N_{0}\right)\right)$ is a martingale. When the orthogonality condition is applied to general optional processes, called $\left\{\Phi_{t}(x), \Psi_{t}(y)\right\}$, the martingale property of $\left\{\Phi_{t}(x) \Psi_{t}(y)\right\}$ is no more equivalent to that of $\left.\left\{\left(\Phi_{t}(x)-x\right)\left(\Psi_{t}(y)-y\right)\right)\right\}$.

In Theorem 2.2, we introduce the assumption "the product of the processes $\Phi_{t}(x)=X_{t}(x)$ and $\Psi_{t}(y)=$ $Y_{t}(y)$ are supermartingales for any $(x, y)>0$ ". This property can be interpreted as a suborthogonality property, depending of the initial conditions.

Definition 2.1 (Different notions of orthogonality). Let $(\boldsymbol{\Phi}, \boldsymbol{\Psi})$ be two optional non negative random fields, and $(v(z))$ be a non negative function. Then, we are concerned with the following notions, 


$$
\left\{\begin{array}{lll}
\text { strong orthogonality : } & \left\{\Phi_{t}(z) \Psi_{t}(y)\right\} \text { is a martingale } \forall(z, y)>0 . \\
\text { v-orthogonality : } & \left\{\Phi_{t}(x) \Psi_{t}(v(x))\right\} \text { is a martingale for any } x>0 . \\
\text { strong suborthogonality : } & \left\{\Phi_{t}(z) \Psi_{t}(y)\right\} \text { is a supermartingale } \forall(z, y)>0 .
\end{array}\right.
$$

Obviously the strong orthogonality implies all the other forms of orthogonality.

As in convex analysis, the set of adapted processes $\left\{Z_{t}\right\}$ which are strongly suborthogonal to a given random field $\left\{\Psi_{t}(y)\right\}$ is a cone, called $\Psi$-suborthogonal cone, (or negative cone) and denoted $\mathcal{Z}^{\Psi}$, where

$$
\mathcal{Z}^{\Psi}=\left\{Z \mid\left\{Z_{t} \Psi_{t}(y)\right\} \text { is a supermartingale } \forall y>0\right\}
$$

\subsubsection{Bi-revealed utility}

All the previous results concern conditions under which a compatible utility is a revealed utility, in terms of $(u, X, Y)$ where the adjoint process $Y$ appears as an auxiliary process. When we are looking for a bi-revealed utility, we only have to add to the conditions of Proposition 2.2 the property that the supermartingale $\left\{X_{t}(x) Y_{t}\left(u_{z}(x)\right)\right\}$ is a martingale for any $x$. In other words, the processes $(X, Y)$ are $u_{z}$-orthogonal. The adjoint process has a nice interpretation in term of Fenchel transform, and conjugate utility function $\widetilde{\mathbf{U}}$. As observed in Definition $1.3, \widetilde{\mathbf{U}}$ is revealed by $Y$ if and only if this martingale property is satisfied.

Theorem 2.2. Let $(u, X, Y) \in \mathcal{A}$ be an admissible triplet generating the compatible utilities $(\mathbf{U}, \widetilde{\mathbf{U}})$. Then, by construction $U_{z}\left(t, X_{t}(x)\right)=Y_{t}\left(u_{z}(x)\right)$.

(i) Assume that $(X, Y)$ are strongly suborthogonal, and $u_{z}$-orthogonal, that is

$$
\left\{\begin{array}{l}
\forall(x, y), \quad\left\{X_{t}(x) Y_{t}(y)\right\} \quad \text { is a supermartingale, } \\
\forall x, \quad\left\{X_{t}(x) Y_{t}\left(u_{z}(x)\right)\right\} \quad \text { is a martingale. }
\end{array}\right.
$$

The utility $\mathbf{U}$ is bi-revealed by the triplet $(u, X, Y)$.

Definition 2.2 (The $\mathcal{A Z}$ set). The processes $(u, X, Y, \mathbb{P}) \in \mathcal{A}$ are said in $\mathcal{A Z}$ if $(X, Y)$ are strongly suborthogonal and $u_{z}$-orthogonal. In particular for any $x,\left\{X_{t}(x)\right\}$ belongs to $\mathcal{Z}^{Y}$.

The description of the elements of the $\mathcal{A Z}$ set is not an easy task.

\subsubsection{Intrinsic universe}

In the bi-revealed case, a very natural hypothesis on the behaviors at the boundary $(0, \infty)$ of the reference processes allows us to make significant progress in this description. The asymptotic in 0 for $X_{t}(x)$, and in $\infty$ for $Y_{t}(y)$ have not been taken into account so far; we are interested in that their behaviors remain close to the deterministic case. The new system, defined as in Section 1.1.4 is called an intrinsic dilated system.

Assumption 2.1 (Limit Assumption).

$$
\text { (Limit Condition) }\left\{\begin{array}{l}
\lim _{x \rightarrow 0} \frac{x_{t}(x)}{x}=\Lambda_{t}^{x}>0 \quad \text { and } \lim _{y \rightarrow \infty} \frac{Y_{t}(y)}{y} \rightarrow H_{t}^{Y}>0 \\
L_{t}^{\text {int }}=\Lambda_{t}^{x} H_{t}^{Y}, L_{0}^{\text {int }}=1 \quad \text { is a } \mathbb{P} \text {-martingale. }
\end{array}\right.
$$

A system $\left\{\left(u, X_{t}(x), Y_{t}(y), \mathbb{P}\right) \in \mathcal{A Z}\right\}$ satisfying the "limit condition" 2.1 is said in $\mathcal{A} \mathcal{Z}^{\text {int }}$.

The martingale property is justified by passing to the limit in $x=0$ in the family of martingales $\left\{X_{t}(x) Y_{t}\left(u_{z}(x)\right) / x u_{z}(x)\right\}$. Rigorous arguments will be given in the next section. 
Theorem 2.3. Let us consider a bi-revealed system $\left\{\left(u, X_{t}(x), Y_{t}(y), \mathbb{P}\right) \in \mathcal{A} \mathcal{Z}^{\text {int }}\right\}$ with limit condition 2.1. and intrinsic system defined by

$$
d \mathbb{Q}^{\text {int }}=L_{T}^{\text {int }} \cdot d \mathbb{P}, \quad x_{t}^{\text {int }}(x)=X_{t}(x) / \Lambda_{t}^{x}, \quad Y_{t}^{\text {int }}(y)=Y_{t}(y) / H_{t}^{Y} .
$$

- The intrinsic system $\left\{\left(u, X_{t}^{\mathrm{int}}(x), Y_{t}^{\mathrm{int}}(y)\right), \mathbb{Q}^{\mathrm{int}}\right\}$ belongs to $\mathcal{A} \mathcal{Z}^{\mathrm{int}}\left(\mathbb{Q}^{\mathrm{int}}\right)$ and is bi-revealed under $\mathbb{Q}^{\mathrm{int}}$.

- More important, for any $(x, y)$, the processes $\left\{X_{t}^{\mathrm{int}}(x)\right\}$, and $\left\{Y_{t}^{\mathrm{int}}(y)\right\}$ are $\mathbb{Q}^{\text {int }}$-supermartingales.

Proof. Thanks to the definition of the intrinsic system, the suborthogonality properties are conserved by change of probability measure and change of numeraire. The first point is then obvious.

The second point asks to be justified. By definition of the family $\mathcal{A Z}$, for a fixed $x,\left\{\mathcal{X}_{t}(x) Y_{t}(y) / y\right\}$ is a $\mathbb{P}$-supermartingale, for any $y$, and this property passes to the limit under some regularity assumption. By Assumption 2.1, letting $y$ goes to $\infty,\left\{X_{t}(x) H_{t}^{Y}\right\}$ is a supermartingale. The same argument shows that $\left\{Y_{t}(y) \Lambda_{t}^{X}\right\}$ is a supermartingale. Since the process $\left\{L_{t}^{\mathrm{int}}=\Lambda_{t}^{X} H_{t}^{Y}\right\}$ is a positive martingale, under the new probability measure $\mathbb{Q}^{\text {int }}=L^{\text {int }} . \mathbb{P}$, the processes $X_{t}^{\text {int }}(x)=X_{t}(x) / \Lambda_{t}^{X}$ and $Y_{t}^{\text {int }}(y)=Y_{t}(y) / H_{t}^{Y}$ are $\mathbb{Q}^{\text {int }}$-supermartingales.

Remark . These supermartingale properties are coherent with the intuition that in expectation, the quality of the information given by the different processes deteriorates over the time.

More important, this result shows clearly that not all monotonic processes can be a characteristic process of a bi-revealed utility problem.

\subsection{An Itô semimartingale framework}

In many dynamic situations, the characteristic process is modeled as an Itô process depending of a parameter, whose local coefficients are known (for instance after calibration from a set of historical data). The same assumption is made on the adjoint process. This is required to be able to propose an algorithm for the construction of the revealed utility. Then, the questions we face are of several types, concerning the elements of the admissible triplet $(u, X, Y) \in \mathcal{A Z}$ :

- a family of purely mathematical problems, to ensure that the basic properties of the characteristic process $X_{t}(x)$ and the adjoint process $Y_{t}(y)$ are satisfied: monotonicity, derivability... The problems are solved thanks to fairly strong regularity hypotheses on the functional coefficients. Definitions and properties are gathered in Section 3.1, a more complete version can be found in [EKM13.

- another family of mathematical questions concerning the dynamics of the compatible utility, defined from the first order condition $U_{z}\left(t, X_{t}(x)\right)=Y_{t}\left(u_{z}(x)\right)$ : is this utility an Itô random field ? If yes, what to say about its functional coefficients, their regularity, the associated SPDE.

The strategy is to focus on the dynamics of the utility of the characteristic process, $\left\{U\left(t, X_{t}(x)\right)\right\}$ which is a martingale for revealed utility. To carry out the calculations, we use an extension of the classical Itô formula for deterministic functions, the Itô-Ventzel formula for regular random field.

\subsubsection{Some notations and calculations}

On the filtered probability space a $d$-dimensional Brownian motion $W$ is defined; $a . b$ denotes the scalar product between two vectors $a$ and $b$. For any Itô-random field $\Phi(t, z)$, we denote by $\left(\beta^{\Phi}(t, z), \gamma^{\Phi}(t, z)\right)$, the functional coefficients, such that

$$
d \Phi(t, z)=\beta^{\Phi}(t, z) d t+\gamma^{\Phi}(t, z) \cdot d W_{t} .
$$

$\beta^{\Phi}(t, z)$ is called the functional trend, and $\gamma^{\Phi}(t, z) \in \mathbb{R}^{d}$ is called the functional diffusion coefficient. We are concerned by the composition of random fields, viewed as an extension of the classical Itô formula. Regularity assumptions are required on the coefficients. 
ITÔ-VENTZEL Formula Let $F(t, z)$ be a regular 11 random field with functional coefficients $\left(\beta^{F}(t, z), \gamma^{F}(t, z)\right)$, such that for any Itô semimartingale $Z, F\left(t, Z_{t}\right)$ is also an Itô semimartingale. Itô-Ventzel's formula gives the semimartingale decomposition of $F\left(t, Z_{t}\right)$.

Proposition 2.3. Let $\{F(t, z)\}$ be a regular random field with functional coefficients $\left(\gamma^{F}, \beta^{F}\right)$, such that $F(t, z)$ and $F_{z}(t, z)$ are linked by

$$
d F(t, z)=\beta^{F}(t, z) d t+\gamma^{F}(t, z) \cdot d W_{t}, \text { and } d F_{z}(t, z)=\beta_{z}^{F}(t, z) d t+\gamma_{z}^{F}(t, z) \cdot d W_{t}
$$

For simplified writing, we put: $\left.\quad d_{t} F(t, z)\right|_{z=Z_{t}}=\beta^{F}\left(t, Z_{t}\right) d t+\gamma^{F}\left(t, Z_{t}\right) . d W_{t}$.

Then, Itô-Ventzel's formula states that $F\left(t, Z_{t}\right)$ is a semimartingale, whose Itô differential is,

$$
d F\left(t, Z_{t}\right)= \begin{cases}\left.d_{t} F(t, z)\right|_{z=Z_{t}}+F_{z}\left(t, Z_{t}\right) d Z_{t}+\frac{1}{2} F_{z z}\left(t, Z_{t}\right)\left\langle d Z_{t}\right\rangle+\left\langle d F_{z}\left(t, Z_{t}\right), d Z_{t}\right\rangle,(\mathrm{USUAL}) \\ \left.d_{t} F(t, z)\right|_{z=Z_{t}}+d\left(F_{z}\left(t, Z_{t}\right) Z_{t}\right)-Z_{t} d F_{z}\left(t, Z_{t}\right)-\frac{1}{2} F_{z z}\left(t, Z_{t}\right)\left\langle d Z_{t}\right\rangle . & \text { (NEW) }\end{cases}
$$

The diffusion term of the semimartingale $F_{z}\left(t, Z_{t}\right)$ is $\left(\gamma_{z}^{F}\left(t, Z_{t}\right)+F_{z z}\left(t, Z_{t}\right) \gamma_{t}^{Z}\right)$.

The first line recalls the classical form of the decomposition when the second line gives a modified version better suited for future calculations.

Proof. The classical Itô-Ventzel formula is the sum of three terms: $d F\left(t, Z_{t}\right)=\left.d_{t} F(t, z)\right|_{z=Z_{t}}+d H_{t}+d K_{t}$. The first term is the "time" differential one, $\left.d_{t} F(t, z)\right|_{z=Z_{t}}=\gamma^{F}\left(t, Z_{t}\right) \cdot d W_{t}+\beta^{F}\left(t, Z_{t}\right) d t$, the second term is similar to a part of the classical Itô formula, $d H_{t}=F_{z}\left(t, Z_{t}\right) d Z_{t}+\frac{1}{2} F_{z z}\left(t, Z_{t}\right)\left\langle d Z_{t}\right\rangle$ and the third one is of covariance type, $d K_{t}=\left\langle d F_{z}\left(t, Z_{t}\right), d Z_{t}\right\rangle$.

This last term can be made more explicit by using the dynamics of $F_{z}\left(t, Z_{t}\right)$, since, under regularity assumptions, $d F_{z}\left(t, Z_{t}\right)=\gamma_{z}^{F}\left(t, Z_{t}\right) \cdot d W_{t}+F_{z z}\left(t, Z_{t}\right) d Z_{t}+\ldots d t$.

The diffusion term of the semimartingale $F_{z}\left(t, Z_{t}\right)$ is $\left(\gamma_{z}^{F}\left(t, Z_{t}\right)+F_{z z}\left(t, Z_{t}\right) \gamma_{t}^{Z}\right)$. So that,

$$
d K_{t}=\left\langle d F_{z}\left(t, Z_{t}\right), d Z_{t}\right\rangle=\left.\left\langle d F_{z}(t, z), d Z_{t}\right\rangle\right|_{z=Z_{t}}+F_{z z}\left(t, Z_{t}\right) d\left\langle Z_{t}\right\rangle
$$

But the quantity $d K_{t}$ also appears in the differential of the product $F_{z}\left(t, Z_{t}\right) . Z_{t}$,

$$
d\left(F_{z}\left(t, Z_{t}\right) Z_{t}\right)=F_{z}\left(t, Z_{t}\right) d Z_{t}+Z_{t} d F_{z}\left(t, Z_{t}\right)+d K_{t}
$$

Coming back to the differential $d F\left(t, Z_{t}\right)-\left.d_{t} F(t, z)\right|_{z=Z_{t}}=d H_{t}+d K_{t}$, we have

$$
d H_{t}+d K_{t}=d\left(F_{z}\left(t, Z_{t}\right) Z_{t}\right)-Z_{t} d F_{z}\left(t, Z_{t}\right)-\frac{1}{2} F_{z z}\left(t, Z_{t}\right) d\left\langle Z_{t}\right\rangle
$$

So we get the new formulation.

\subsubsection{Bi-revealed utilities}

Equipped with this new formulation of the Itô-Ventzel formula, we are able to briefly describe the dynamics of the revealed utility issued from the admissible triplet $(u, X, Y) \in \mathcal{A Z}$ where the processes $\left\{X_{t}(x)\right\}$ and $\left\{Y_{t}(y)\right\}$ are Itô processes satisfying the orthogonality constraints of Theorem 2.2 " $\left\{\mathcal{X}_{t}(x) Y_{t}\left(u_{z}(x)\right)\right\}$ is martingale and $\left\{\mathcal{X}_{t}(x) Y_{t}(y)\right\}$ are supermartingales for any $(x, y)$ ".

By classical Itô calculus, the strong suborthogonality condition is equivalent to

$$
\text { Strong Sub-Ortho: } \quad Y_{t}(y) \beta_{t}^{x}(x)+x_{t}(x) \beta^{Y}(t, y)+\left\langle\gamma^{x}(t, x), \gamma^{Y}(t, y)\right\rangle \leq 0, \quad \forall(x, y) .
$$

\footnotetext{
${ }^{1} F$ is of class $\mathcal{C}^{2}$ in $z$ with $\partial_{z}^{2} F$ is $\delta$-Hölder for some $\delta>0$. This implies, in particular, that $\left(\beta^{F}\right.$ and $\left.\gamma^{F}\right)$ are of class $C^{2}$ with $\beta_{z z}^{F}:=\partial_{z}^{2} \beta^{F}$ and $\gamma_{z z}^{F}:=\partial_{z}^{2} \gamma^{F}$ are $\varepsilon$-Hölder for any $0<\varepsilon<\delta$, see [EKM13] or [Kun97.
} 
The functional coefficients of the compatible utility (assumed to be an Itô random field) $\left(\beta^{U}(t, z), \gamma^{U}(t, z)\right.$ ) are assumed sufficiently regular so that the functional coefficients of the marginal utility are the derivatives of those of $U$. The functional coefficients of the processes $\left(\mathcal{X}_{t}(x), Y_{t}(y)\right)$ will be made explicitly only when we need them. More on regularity issues can be found in Section 4 and in details in EKM13.

Theorem 2.4 (Bi-Revealed Utility coefficients). Assume $\mathbf{U}$ be a regular random field $U$, bi-revealed by $(u, X, Y) \in \mathcal{A Z}$. The random fields $(X, Y)$ are assumed to be regular Itô processes, such that

$$
d U(t, z)=\beta^{U}(t, z) d t+\gamma^{U}(t, z) \cdot d W_{t}, \quad d U_{z}(t, z)=\beta_{z}^{U}(t, z) d t+\gamma_{z}^{U}(t, z) \cdot d W_{t}
$$

Then, the first order condition $U_{z}\left(t, X_{t}(x)\right)=Y_{t}\left(u_{z}(x)\right)$ and the martingale property of the processes $\left\{U\left(t, X_{t}(x)\right)\right\}$ and $\left\{X_{t}(x) Y_{t}\left(u_{z}(x)\right)\right\}$ imply

$$
\left\{\begin{array}{l}
d U\left(t, x_{t}(x)\right)=\gamma^{U}\left(t, x_{t}(x)\right) \cdot d W_{t}+d\left(U_{z}\left(t, x_{t}(x)\right) X_{t}(x)\right)-x_{t}(x) \gamma^{Y}\left(t, u_{z}(x)\right) \cdot d W_{t}, \\
\beta^{U}\left(t, x_{t}(x)\right) d t=\frac{1}{2} U_{z z}\left(t, x_{t}(x)\right) d\left\langle X_{t}(x)\right\rangle+x_{t}(x) \beta^{Y}\left(t, u_{z}(x)\right) d t \\
\gamma_{z}^{U}\left(t, x_{t}(x)\right)=\gamma^{Y}\left(t, u_{z}(x)\right)-U_{z z}\left(t, x_{t}(x)\right) \gamma^{x}(t, x) .
\end{array}\right.
$$

Remark that the identification of the diffusion coefficient $\gamma_{z}^{U}\left(t, X_{t}(x)\right)$ of $U_{z}\left(t, X_{t}(x)\right)$ doesn't use any martingale assumption.

Proof. The proof is a direct application of the new version of the Itô-Ventzel formula, applied to $F(t, z)=$ $U(t, z)$ and $Z_{t}=X_{t}(x)$, we have,

$$
\begin{aligned}
d U\left(t, X_{t}(x)\right) & =\gamma^{U}\left(t, x_{t}(x)\right) \cdot d W_{t}+d\left(U_{z}\left(t, X_{t}(x)\right) X_{t}(x)\right) \\
& +\beta^{U}\left(t, X_{t}(x)\right) d t-\frac{1}{2} U_{z z}\left(t, X_{t}(x)\right)\left\langle d X_{t}(x)\right\rangle-X_{t}(x) d Y_{t}\left(u_{z}(x)\right) .
\end{aligned}
$$

The martingale property of the two processes $\left.\left\{U_{z}\left(t, X_{t}(x)\right) X_{t}(x)=Y_{t}\left(u_{z}(x)\right) X_{t}(x)\right)\right\}$ and $\left\{U\left(t, X_{t}(x)\right)\right\}$ implies that $d N_{t}=\beta^{U}\left(t, X_{t}(x)\right) d t-\frac{1}{2} U_{z z}\left(t, X_{t}(x)\left\langle d X_{t}(x)\right\rangle-X_{t}(x) d Y_{t}\left(u_{z}(x)\right)\right.$ is the differential of a martingale; its finite variation term is identical to 0 , which allows the identification of $\beta^{U}\left(t, \chi_{t}(x)\right)$ given in the theorem.

The coefficient $\gamma_{z}^{U}\left(t, X_{t}(x)\right)$ has been identified in the proof of Proposition 2.3 from the relation

$$
d U_{z}\left(t, X_{t}(x)\right)=d Y_{t}\left(u_{z}(x)\right)=\gamma_{z}^{U}\left(t, X_{t}(x)\right) \cdot d W_{t}+U_{z z}\left(t, X_{t}(x)\right) d X_{t}(x)+\ldots d t
$$

The third relation is a simple consequence of this observation.

The dynamics of the Fenchel conjugate $\widetilde{\mathbf{U}}$ of $\mathbf{U}$ is obtained easily from the previous calculation. The key property is once again the master formula, since $\tilde{U}\left(t, Y_{t}\left(u_{z}(x)\right)\right)=U\left(t, X_{t}(x)\right)-X_{t}(x) Y_{t}\left(u_{z}(x)\right)$. This identity shows that $\widetilde{U}\left(t, Y_{t}\left(u_{z}(x)\right)\right)$ is a martingale, confirming that $\widetilde{\mathbf{U}}$ is a conjugate utility revealed by $\left\{\tilde{u}, Y_{t}(y)\right\}$. Moreover, the regularity assumptions made on the processes $(\mathcal{X}, Y)$ to bi-revealate $\mathbf{U}$ ensure that $\widetilde{\mathbf{U}}$ is a regular semimartingale, whose characteristics are denoted by $\beta^{\widetilde{U}}$ and $\gamma^{\widetilde{U}}$.

Proposition 2.4. Under the hypothesis of Theorem 2.4, with the short notation $x(y)=-\tilde{u}_{y}(y)$, and $\left.x_{t}(x(y))\right)=-\widetilde{U}_{y}\left(t, Y_{t}(y)\right)$, we have

$$
\left\{\begin{array}{l}
d \widetilde{U}\left(t, Y_{t}(y)\right)=\gamma^{U}\left(t, x_{t}(x(y))\right) \cdot d W_{t}-d\left(\widetilde{U}_{y}\left(t, Y_{t}(y)\right) Y_{t}(y)\right)+\widetilde{U}_{y}\left(t, Y_{t}(y)\right) \gamma^{Y}(t, y) \cdot d W_{t} \\
\gamma^{\widetilde{U}}\left(t, Y_{t}(y)\right)=\gamma^{U}\left(t,-\widetilde{U}_{y}\left(t, Y_{t}(y)\right)\right) \\
\beta^{\widetilde{U}}\left(t, Y_{t}(y)\right) d t=\frac{1}{2} \widetilde{U}_{y y}\left(t, Y_{t}(y)\right)\left\langle d Y_{t}(y)\right\rangle-Y_{t}(y) \beta^{x}(t, x(y)) d t
\end{array}\right.
$$

The first equation establishes as expected, that $\left\{\widetilde{U}\left(t, Y_{t}(y)\right)\right\}$ is a martingale. 
Proof. We start from the identity $\widetilde{U}\left(t, Y_{t}\left(u_{z}(x)\right)\right)=U\left(t, X_{t}(x)\right)-X_{t}(x) Y_{t}\left(u_{z}(x)\right)$ and $X_{t}(x(y))=-\widetilde{U}_{y}\left(t, Y_{t}(y)\right)$, and the formula

$$
\begin{aligned}
& d \widetilde{U}\left(t, Y_{t}\left(u_{z}(x)\right)\right)=d U\left(t, X_{t}(x)\right)-d\left(U_{z}\left(t, X_{t}(x)\right) X_{t}(x)\right) \\
& =\gamma^{U}\left(t, X_{t}(x)\right) \cdot d W_{t}+\beta^{U}\left(t, X_{t}(x)\right) d t-\frac{1}{2} U_{z z}\left(t, X_{t}(x)\right)\left\langle d X_{t}(x)\right\rangle-X_{t}(x) d Y_{t}\left(u_{z}(x)\right) .
\end{aligned}
$$

On the other hand, the Itô-Ventzel formula gives,

$$
\begin{aligned}
d \widetilde{U}\left(t, Y_{t}\left(u_{z}(x)\right)\right) & =\gamma^{\widetilde{U}}\left(t, Y_{t}\left(u_{z}(x)\right)\right) \cdot d W_{t}+\beta^{\widetilde{U}}\left(t, Y_{t}\left(u_{z}(x)\right)\right) d t+\frac{1}{2} \widetilde{U}_{y y}\left(t, Y_{t}\left(u_{z}(x)\right)\right)\left\langle d Y_{t}\left(u_{z}(x)\right)\right\rangle \\
& -x_{t}(x) d Y_{t}\left(u_{z}(x)\right)+\left\langle\gamma_{y}^{\widetilde{U}}\left(t, Y_{t}\left(u_{z}(x)\right)\right), \sigma_{t}^{Y}\left(Y_{t}\left(u_{z}(x)\right)\right)\right\rangle .
\end{aligned}
$$

By identifying the terms, as for the dynamics of $\mathbf{U}$, and by using the suborthogonality condition and the fact that $X_{t}\left(-\tilde{u}_{y}(y)\right)=-\widetilde{U}_{y}\left(t, Y_{t}(y)\right)$ and $-\widetilde{U}_{y y}\left(Y_{t}\left(u_{z}(x)\right)\right) U_{z z}\left(t, X_{t}(x)\right)=1$, yields to the equation of the proposition, where $\tilde{\xi}$ denotes the inverse of $Y$.

In the intrinsic case studied in Section 2.1.5 interesting properties can be immediately deduced on the revealed utility and its conjugate.

Corollary 2.5. Assume an intrinsic universe, where the processes $\left\{X_{t}^{\mathrm{int}}(x)\right\}$ and $\left\{Y_{t}^{\mathrm{int}}(y)\right\}$ are $\mathbb{Q}^{\mathrm{int}}$ supermartingales. Then, the revealed utility $\left\{U^{\mathrm{int}}(t, z)\right\}$ is a $\mathbb{Q}^{\mathrm{int}}$-supermartingale and the conjugate utility $\left\{\widetilde{U}^{\text {int }}(t, y)\right\}$ is a $\mathbb{Q}^{\text {int }}$-submartingale.

Proof. The proof relies on the identification of the drift random fields of the revealed utility function and its conjugate

$$
\left\{\begin{array}{l}
\beta^{U, i n t}\left(t, X_{t}^{\mathrm{int}}(x)\right) d t=\frac{1}{2} U_{z z}^{\mathrm{int}}\left(t, X_{t}^{\mathrm{int}}(x)\right) d\left\langle X_{t}^{\mathrm{int}}(x)\right\rangle+X_{t}^{\mathrm{int}}(x) \beta^{Y, \mathrm{int}}\left(t, u_{z}(x)\right) d t, \\
\beta^{\widetilde{U}, \text { int }}\left(t, Y_{t}^{\mathrm{int}}(y)\right) d t=\frac{1}{2} \widetilde{U}_{y y}^{\mathrm{int}}\left(t, Y_{t}^{\mathrm{int}}(y)\right)\left\langle d Y_{t}^{\mathrm{int}}(y)\right\rangle-Y_{t}^{\mathrm{int}}(y) \beta^{X, \mathrm{int}}(t, x(y)) d t
\end{array}\right.
$$

The supermartingale properties of the processes $\left\{X_{t}^{\text {int }}(x)\right\}$ and $\left\{Y_{t}^{\text {int }}(y)\right\}$ imply that their intrinsic drifts $\beta^{X, \text { int }}(t, x(y)) d t$ and $\beta^{Y, \text { int }}\left(t, u_{z}(x)\right) d t$ are negative. Thanks to the concavity of $U^{\text {int }}(t, z), U_{z z}^{\text {int }}(t, z)$ is negative, and $\beta^{U, \text { int }}\left(t, X_{t}(x)\right)$ is decreasing. Then, $\beta^{U, \text { int }}(t, z)$ is negative. Similar argument can be used for the conjugate utility, since $\beta^{x, \text { int }}(t, x(y))$ is negative and $\widetilde{U}_{y y}^{\text {int }}\left(t, Y_{t}^{\text {int }}(y)\right)$ is positive by convexity of $\widetilde{U}^{\text {int }}(t, y)$. Then $\beta^{\widetilde{U}, \text { int }}\left(t, Y_{t}^{\text {int }}(y)\right)$ is increasing and $\beta^{\widetilde{U}, i n t}(t, y)$ is positive.

This result generalizes the notion of decreasing forward utility introduced in financial market by Musiela and Zariphopoulo in [MZ10a].

SDEs framework The description of the differential coefficients $\left(\beta^{U}(t, z), \gamma_{z}^{U}(t, z)\right)$ can be made more explicit if the dynamics of the generating processes $X_{t}(x)$ and $Y_{t}(y)$ are given as a stochastic differential equations with stochastic coefficients, whose properties are explained in Section 3.1 .

$$
\begin{cases}\gamma^{x}(t, x)=\sigma_{t}^{x}\left(X_{t}(x)\right), & \gamma^{Y}(t, y)=\sigma_{t}^{Y}\left(Y_{t}(y)\right) \\ \beta^{x}(t, x)=\mu_{t}^{x}\left(X_{t}(x)\right), & \beta^{Y}(t, y)=\mu_{t}^{Y}\left(Y_{t}(y)\right)\end{cases}
$$

In this case, we can better specify the constraints on the coefficients $\left(\sigma_{t}^{x}(z), \mu_{t}^{x}(z)\right)$ and $\left(\sigma_{t}^{Y}(y), \mu_{t}^{Y}(y)\right)$ so that the properties of Theorem 2.2 are fulfilled. These constraints, referenced as the $\mathcal{A} Z$ condition in Definition 2.2, should therefore reflect the fact that $X_{t}(z) Y_{t}(y)$ is a supermartingale for any $(z, y)$ and a martingale for $y=u_{z}(x)$.

$$
(\mathcal{A} Z \text { - condition })\left\{\begin{array}{l}
z \mu_{t}^{Y}(y)+y \mu_{t}^{X}(z)+\left\langle\sigma_{t}^{x}(z), \sigma_{t}^{Y}(y)\right\rangle \leq 0, \forall(z, y) a . s \\
z \mu_{t}^{Y}\left(U_{z}(t, z)\right)+U_{z}(t, z) \mu_{t}^{x}(z)+\left\langle\sigma_{t}^{x}(z), \sigma_{t}^{Y}\left(U_{z}(t, z)\right)\right\rangle=0, a . s .
\end{array}\right.
$$


The case of intrinsic universe, defined in Section 2, simplifies the interpretation.

Proposition 2.5. Let the processes $X_{t}(z)$ and $Y_{t}(y)$ satisfy the $\mathcal{A} Z$-condition.

The differential coefficients of the revealed utility $\mathbf{U}$ are :

$$
\left(\mathrm { U } \text { - coefficients) } \left\{\begin{array}{l}
\beta^{U}(t, z)=\frac{1}{2} U_{z z}(t, z)\left\|\sigma_{t}^{x}(z)\right\|^{2}+z \mu_{t}^{Y}\left(U_{z}(t, z)\right) \\
\gamma_{z}^{U}(t, z)=\sigma_{t}^{Y}\left(U_{z}(t, z)\right)-U_{z z}(t, z) \sigma_{t}^{x}(z)
\end{array}\right.\right.
$$

When $\left\{Y_{t}(y)\right\}$ is a supermartingale, as in the intrisic case, $\{U(t, z)\}$ is also a super martingale.

Remark . The decomposition of $\beta^{U}$ is not unique since, from the orthogonality constraints (2.5),

$$
\left\{\begin{array}{l}
\beta^{U}(t, z)=\frac{1}{2} U_{z z}(t, z)\left\|\sigma_{t}^{x}(z)\right\|^{2}-U_{z}(t, z) \mu_{t}^{x}(z)-\left\langle\sigma_{t}^{x}(z), \sigma_{t}^{Y}\left(U_{z}(t, z)\right)\right\rangle, \\
\beta^{U}(t, z)+\left\langle\gamma_{z}^{U}(t, z), \sigma_{t}^{x}(z)\right\rangle=-\left[\frac{1}{2} U_{z z}(t, z)\left\|\sigma_{t}^{x}(z)\right\|^{2}+U_{z}(t, z) \mu_{t}^{x}(z)\right] .
\end{array}\right.
$$

Proof. - The first condition relies on the drift coefficient $K_{t}(x, y)$ of the semimartingale $\left\{X_{t}(x) Y_{t}(y)\right\}$, $K_{t}(x, y)=X_{t}(x) \mu_{t}^{Y}\left(Y_{t}(y)\right)+Y_{t}(y) \mu^{X}\left(t, X_{t}(x)\right)+\left\langle\sigma_{t}^{X}\left(X_{t}(x)\right), \sigma_{t}^{Y}\left(Y_{t}(y)\right)\right\rangle=k_{t}\left(X_{t}(x), Y_{t}(y)\right)$. The first supermartingale condition is equivalent to $K_{t}(x, y) \leq 0, \forall(x, y)$. Since the processes are invertible, with range $[0, \infty)$, the condition can be given directly in terms of coefficients as $k_{t}(x, y) \leq 0$.

- When $y=u_{z}(z)$, the same transformation uses that $Y_{t}\left(u_{z}(z)\right)=U_{z}\left(t, X_{t}(z)\right)$, and the martingale property is given by the second equality, which is depending of the marginal utility $U_{z}(t, z)$.

\subsection{Revealed utility and optimization}

Generally, utility criteria are associated with optimization problem. But, this is not the case here, given the definition of revealed utilities problem. However, under the $\mathcal{A} Z$-conditions of Theorem 2.2, based on the strong suborthogonality of the processes $(X, Y)$, the cone of strongly suborthogonal processes $\mathcal{Z}^{Y}$ defined in 2.2 appears to be a natural set for test processes $Z$ whose performance $U\left(t, Z_{t}\right)$ is expected to be a supermartingale.

\subsubsection{Differential Utility property and $Y$-strong suborthogonality}

The utility framework to which we shall refer satisfies the $\mathcal{A} Z$-condition, which implies that

$$
\begin{aligned}
& -\beta^{U}(t, z)=\frac{1}{2} U_{z z}(t, z)\left\|\sigma_{t}^{x}(z)\right\|^{2}+z \mu_{t}^{Y}\left(U_{z}(t, z)\right) \\
& -\gamma_{z}^{U}(t, z)=\sigma_{t}^{Y}\left(U_{z}(t, z)\right)-U_{z z}(t, z) \sigma_{t}^{X}(z)
\end{aligned}
$$

Let $\left\{Z_{t}\right\}$ be an Itô semimartingale with dynamics $d Z_{t}=\phi_{t}^{Z} d t+\psi_{t}^{Z} \cdot d W_{t}$, described by its parameters $\left(\phi_{t}^{Z}, \psi_{t}^{Z}\right)$. From Itô-Ventzel's formula, the $U$-performance of $\left\{Z_{t}\right\}$ is a semimartingale whose dynamics is easily computable.

Proposition 2.6. Let $(u, X, Y) \in \mathcal{A Z}$ be an admissible triplet generating the bi-revealed dynamic utility $\mathbf{U}$ with the previous differential properties. Then, for any semimartingale $\left\{Z_{t}\right\}$ with parameters $\left(\phi_{t}^{Z}, \psi_{t}^{Z}\right)$

$$
\begin{array}{r}
d U\left(t, Z_{t}\right)=\frac{1}{2} U_{z z}\left(t, Z_{t}\right)\left\|\sigma_{t}^{X}\left(Z_{t}\right)-\psi_{t}^{Z}\right\|^{2} d t+\left(\gamma^{U}\left(t, Z_{t}\right)+U_{z}\left(t, Z_{t}\right) \psi_{t}^{Z}\right) d W_{t} \\
+\left[Z_{t} \mu_{t}^{Y}\left(U_{z}\left(t, Z_{t}\right)\right)+U_{z}\left(t, Z_{t}\right) \phi_{t}^{Z}+\left\langle\sigma_{t}^{Y}\left(U_{z}\left(t, Z_{t}\right)\right), \psi_{t}^{Z}\right\rangle\right] d t
\end{array}
$$

The last term is the drift of the product $\left\{Z_{t} Y_{t}(y)\right\}$ taken at $y=U_{z}\left(t, Z_{t}\right)$. 
Proof. The Itô-Ventzel formula applied to the random field $\{U(t, z)\}$ and to the semimartingale $\left\{Z_{t}\right\}$ implies

$$
\left\{\begin{array}{l}
d U\left(t, Z_{t}\right)=\gamma^{U}\left(t, Z_{t}\right) \cdot d W_{t}+U_{z}\left(t, Z_{t}\right) \psi_{t}^{Z} \cdot d W_{t} \\
+\beta^{U}\left(t, Z_{t}\right)+\left\langle\gamma_{z}^{U}\left(t, Z_{t}\right), \psi_{t}^{Z}\right\rangle+U_{z}\left(t, Z_{t}\right) \phi_{t}^{Z}+\frac{1}{2} U_{z z}\left(t, Z_{t}\right)\left\|\psi_{t}^{Z}\right\|^{2} \\
\left\langle\gamma_{z}^{U}\left(t, Z_{t}\right), \psi_{t}^{Z}\right\rangle=\left\langle\sigma_{t}^{Y}\left(U_{z}\left(t, Z_{t}\right)\right), \psi_{t}^{Z}\right\rangle-U_{z z}\left(t, Z_{t}\right)\left\langle\sigma_{t}^{x}\left(Z_{t}\right), \psi_{t}^{Z}\right\rangle .
\end{array}\right.
$$

From the definition of $\beta^{U}\left(t, Z_{t}\right)=\frac{1}{2} U_{z z}\left(t, Z_{t}\right)\left\|\sigma_{t}^{X}\left(Z_{t}\right)\right\|^{2}+Z_{t} \mu_{t}^{Y}\left(U_{z}\left(t, Z_{t}\right)\right)$, the term in $U_{z z}\left(t, Z_{t}\right)$ in the decomposition of $U\left(t, Z_{t}\right)$ appears with the coefficient $\frac{1}{2}\left\|\sigma_{t}^{X}\left(Z_{t}\right)\right\|^{2}+\frac{1}{2}\left\|\psi_{t}^{Z}\right\|^{2}-\left\langle\sigma_{t}^{X}\left(Z_{t}\right), \psi_{t}^{Z}\right\rangle=$ $\frac{1}{2}\left\|\sigma_{t}^{X}\left(Z_{t}\right)-\psi_{t}^{Z}\right\|^{2}$; by concavity of $\mathbf{U}$, this term is non positive. The remaining drift is given by $K_{t}(Z, Y)=Z_{t} \mu_{t}^{Y}\left(U_{z}\left(t, Z_{t}\right)\right)+U_{z}\left(t, Z_{t}\right) \phi_{t}^{Z}+\left\langle\sigma_{t}^{Y}\left(U_{z}\left(t, Z_{t}\right)\right), \psi_{t}^{Z}\right\rangle$, which is the drift of the product $\left\{Z_{t} Y_{t}(y)\right\}$ taken at time $t$ at $y=U_{z}\left(t, Z_{t}\right)$.

Controlled processes The set of controlled processes is the $Y$-suborthogonal cone $\mathcal{Z}^{Y}$ of Itô processes $\left\{Z_{t}\right\}$, with differential parameters $\left(\phi_{t}^{Z}, \psi_{t}^{Z}\right)$, strongly suborthogonal to $\left\{Y_{t}(y)\right\}$. The space of controls is the family of processes $\left(Z_{t}, \phi_{t}^{Z}, \psi_{t}^{Z}\right)$, with linear constraints

$$
Z_{t}>0, \quad y \phi_{t}^{Z}+Z_{t} \mu_{t}^{Y}(y)+\left\langle\psi_{t}^{Z}, \sigma_{t}^{Y}(y)\right\rangle \leq 0, \quad \forall y, \text { a.s. }
$$

This set contains at least the family $\left\{X_{t}(x)\right\}$ for any $x$, whose coefficients are $\left(\mu_{t}^{X}\left(X_{t}(x)\right), \sigma_{t}^{X}\left(X_{t}(x)\right)\right)$.

Theorem 2.6. Under the assumptions of Proposition 2.6.

For any control $\left\{\left(Z_{t}, \phi_{t}^{Z}, \psi_{t}^{Z}\right)\right\}$ strong suborthogonal to $\left\{Y_{t}(y)\right\}$,

- $U\left(t, Z_{t}\right)$ is a supermartingale.

- $U\left(t, Z_{t}\right)$ is a martingale if and only if $Z$ is a solution of the $S D E\left(\mu^{x}, \sigma^{x}\right)$, and then, by uniqueness of $S D E\left(\mu^{x}, \sigma^{x}\right), Z_{t}=x_{t}\left(Z_{0}\right)$.

Proof. (i) - Thanks to equation 2.6 in Proposition 2.6, in the decomposition of $U\left(t, Z_{t}\right)$ the term in $U_{z z}\left(t, Z_{t}\right)$ appears with the coefficient $\frac{1}{2}\left\|\sigma_{t}^{X}\left(Z_{t}\right)-\psi_{t}^{Z}\right\|^{2}$; by concavity of $\mathbf{U}$ this term is negative. The remaining drift is given by the drift term of the product $Z_{t} \mu_{t}^{Y}\left(U_{z}\left(t, Z_{t}\right)\right)+U_{z}\left(t, Z_{t}\right) \phi_{t}^{Z}+\left\langle\sigma_{t}^{Y}\left(U_{z}\left(t, Z_{t}\right)\right), \psi_{t}^{Z}\right\rangle$. Since $Z$ is strongly orthogonal to $\left\{Y_{t}(y)\right\}$, this term is also negative, and the process $U\left(t, Z_{t}\right)$ is a supermartingale.

- As the drift term of $U\left(t, Z_{t}\right)$ is the sum of two non positive terms, $U\left(t, Z_{t}\right)$ is a martingale iff

$$
\frac{1}{2} U_{z z}\left(t, Z_{t}\right)\left\|\sigma_{t}^{x}\left(Z_{t}\right)-\psi_{t}^{Z}\right\|^{2}=0, \text { and } Z_{t} \mu_{t}^{Y}\left(Y_{t}(y)\right)+Y_{t}(y) \phi_{t}^{Z}+\left\langle\psi_{t}^{Z}, \sigma_{t}^{Y}\left(Y_{t}(y)\right)\right\rangle=0, \forall y
$$

The first one is equivalent to $\psi_{t}^{Z}=\sigma_{t}^{X}\left(Z_{t}\right)$. This identity injected in the second one and the orthogonality condition of $\left\{X_{t}(x)\right\}$ and $\left\{Y_{t}\left(u_{z}(x)\right)\right\}$ imply

$$
Z_{t} \mu_{t}^{Y}\left(Y_{t}(y)\right)+Y_{t}(y) \phi_{t}^{Z}=Z_{t} \mu_{t}^{Y}\left(Y_{t}(y)\right)+Y_{t}(y) \mu_{t}^{x}\left(Z_{t}\right), \forall y
$$

so that $\phi_{t}^{Z}=\mu_{t}^{x}\left(Z_{t}\right), \forall t, z$. Then, by uniqueness (ensured by Proposition 3.1) of the solution of the $\operatorname{SDE}\left(\mu^{x}, \sigma^{x}\right)$, it follows that $Z_{t}=X_{t}\left(Z_{0}\right), \mathbb{P}$ a.s.

Remark 2.1. As a direct consequence of the previous result, we have that the initial utility is the value function of the optimization problem, for any time-horizon $t$.

$$
U(0, z):=u(z)=\sup _{Z \in \mathcal{Z}^{Y}, Z_{0}=z} \mathbb{E}\left(U\left(t, Z_{t}\right)\right), \forall t \geq 0 .
$$


Using the notion of forward starting problem introduced in Section 1.2.2, Proposition 1.3, the interpretation of the bi-revealed utility as value function of control problem can be generalized to the cone of strongly suborthogonal process starting from $(z, s)$.

$$
\begin{aligned}
& \mathcal{Z}^{Y}(z, s)=\left\{Z \geq 0: Z_{s}=z \text { and }\left\{Z_{t} Y_{t}(y, s)\right\}_{t \geq s} \text { is a supermartingale for any } y\right\} . \\
& \text { Then } U(s, z)=\mathrm{ess} \sup _{Z \in \mathcal{Z}^{Y}(z, s)} \mathbb{E}\left(U\left(t, Z_{t}\right) / \mathcal{F}_{s}\right), \forall t \geq s .
\end{aligned}
$$

Example of e-commerce Problems generated by the e-commerce, see William \& al [WTKD04, belong to this family. The main problem is to build the dynamic utility of a user from his search history and purchases on the Internet, considered as the characteristic process. In e-commerce, the adjoint process $Y$ is used as an indicator of the probability to purchase of the consumer and it is adjusted gradually according to the history of purchases, the prices proposed and the consumer's reaction to them. Once estimated, it is used to learn the consumer utility and the orthogonal cone $\mathcal{Z}^{Y}$ which, in e-commerce, represents the class of products that cannot interest the agent. Therefore, only products or items that are in the complement of $\mathcal{Z}^{Y}$ are attractive to this consumer because they have chances to be bought or chosen since their performance are not declining on average.

\section{Complementary results and the strong orthogonality frame- work.}

In this section, we deepen the framework of Itô semimartingale of Section 2.2, and we provide insights on the consequences of the $\mathcal{A} Z$ condition, in particular the strong suborthogonality (resp. orthogonality) valid for any $x$ and $y$. But before, we recall some results on the regularity of SDE solution.

\subsection{SDE's solutions and regularity}

To be more precise, we suppose that the observable process $X$ and the adjoint process $Y$ are solutions of two stochastic differential equations (SDE) with random coefficients. The existing literature provides explicit conditions ensuring the existence, monotonicity and regularity of the solutions. From these results, it will become easy to deduce the regularity of compatible utility $\mathbf{U}$ to apply assumptions of Section 2.2 . in particular the Itô-Ventzel formula. Similar questions have been considered in [EKM13, with a rigorous treatment of the regularity of the solutions of stochastic differential equations (SDEs) with random coefficients; all references are relative to this paper.

Before recalling general regularity results on one dimensional SDEs, we fix the generic notation for the coefficients and the solutions $X$ and $Y$. The probability space $\left(\Omega,\left(\mathcal{F}_{t}\right), \mathbb{P}\right)$ is equipped with a $d$-dimensional Brownian motion $\left\{W_{t}\right\}$ driving the different SDE's, with random coefficients of type $\left(\mu_{t}(z), \sigma_{t}(z)\right)$,

$$
\left\{\begin{array}{l}
\text { SDE form: } d X_{t}(x)=\mu_{t}\left(X_{t}(x)\right) d t+\sigma_{t}\left(X_{t}(x)\right) \cdot d W_{t}, \quad X_{0}=x \\
\text { Multiplicative decomposition } \quad d X_{t}(x)=X_{t}(x)\left[\rho_{t}\left(X_{t}(x)\right) d t+\kappa_{t}\left(X_{t}(x)\right) \cdot d W_{t}\right], \\
\text { Random field } \quad d X_{t}(x)=\beta(t, x) d t+\gamma(t, x) \cdot d W_{t}, \quad X_{0}=x, \\
\text { Differential parameters } \beta(t, x)=\mu_{t}\left(X_{t}(x)\right), \quad \gamma(t, x)=\sigma_{t}\left(X_{t}(x)\right) .
\end{array}\right.
$$

The second line is only concerned with positive solutions; the third one is an interpretation of the SDE's solution as a random field, with differential parameters $(\beta(t, x), \gamma(t, x))$. In the sequel, the interplay between these different formulations facilitates analysis and interpretations. The SDE point of view is 
better suited to questions relative to the order structure as positivity, monotonicity, concavity, when the random field point of view is better suited to differentiability issues.

\subsubsection{Regularity issues: a synthesis}

In [EKM13, several technical questions were raised and solved in order to carry out the calculations and deduce the dynamics of the compatible utility $\mathbf{U}$ and its dual $\widetilde{\mathbf{U}}$. The classes $\mathcal{K}^{m, \delta}\left(m \in \mathbb{N}^{*}, \delta \in(0,1]\right)$ of random fields played a major role.

Definition 3.1. An optional random field $\left(\phi \in \mathbb{R}^{d}\right)(d \geq 1)$, such that $\phi(t, 0)=0$ a.s., is said to be in the class $\mathcal{K}_{\text {loc }}^{m, \delta}\left(m \in \mathbb{N}^{*}, \delta \in(0,1]\right)$ if $\phi$ is of class $\mathcal{C}^{m}$ in $x$ with continuous,"locally" bounded derivatives, such that $\partial^{m} \phi$ is $\delta$-Hölder. When the derivatives of $\phi$ are bounded, $\phi$ is said to be in the class $\mathcal{K}_{b}^{m, \delta}$.

Let us recall here some results on SDEs useful for our purpose.

Proposition 3.1. Let us consider the $S D E, d X_{t}(x)=\mu_{t}^{X}\left(X_{t}(x)\right) d t+\sigma_{t}^{X}\left(X_{t}(x)\right) \cdot d W_{t}, \quad X_{0}=x$.

i) When $\mu^{X}$ and $\sigma^{X}$ are in the class $\mathcal{K}_{\text {loc }}^{1, \delta}, \delta>0$, a strong solution $X_{t}(x)$ exists, is unique, positive, increasing and regularly differentiable in $x$, with differential

$$
\left.d X_{x}(t, x)=X_{x}(t, x)\left[\mu_{x}^{X}\left(t, X_{t}(x)\right)\right) d t+\sigma_{x}^{X}\left(t, X_{t}(x)\right) \cdot d W_{t}\right], \quad X_{x}(0, x)=1
$$

Moreover, if $\mu^{X}$ and $\sigma^{X}$ are $\mathcal{K}_{l o c}^{m, \delta}$, then $X$ is in the class $\mathcal{K}_{l o c}^{m, \varepsilon}, \delta<\epsilon$.

ii) In particular, under the same assumptions, the function $x \rightarrow X_{t}(x)$ is increasing with range $[0, \infty]$. More precisely, if $\max X_{T}(x)=\sup _{0 \leq t \leq T} X_{t}(x)$,

- When $x$ goes to $\infty$, almost surely, for any $\varepsilon \in(0,1)$, uniformly on $[0, T]$,

$$
\lim _{x \rightarrow+\infty}\left(x^{-(1+\varepsilon)} \max X_{T}(x)\right)=0 \text { and } \lim _{x \rightarrow+\infty}\left(x^{-\varepsilon} \max X_{T}(x)\right)=+\infty, \text { for any } T \text {. }
$$

- When $x$ goes to 0 , almost surely, for any $\varepsilon \in(0,1)$, uniformly on $[0, T]$

$$
\lim _{x \rightarrow 0}\left(x^{-\varepsilon} \max X_{T}(x)\right)=0 \text { and } \lim _{x \rightarrow 0}\left(x^{-(1+\varepsilon)} \max X_{T}(x)\right)=+\infty, \text { for any } T .
$$

iii) Furthemore, if $m \geq 3$ then the inverse flow $\{\xi(t, z)\}$ of $\left(X_{t}(x)\right)$ is a semimartingale random field in the class $\mathcal{K}_{l o c}^{m-1, \varepsilon}, \forall \varepsilon<\delta$.

From regular SDEs to regular utility Let us consider that the characteristic process $\left\{X_{t}(x)\right\}$ and its adjoint $\left\{Y_{t}(y)\right\}$ are regular solutions of some SDE's, generating a compatible marginal utility (with initial $\mathcal{C}^{2}$ utility $\left.u\right)$ by $U_{z}(t, z)=Y_{t}\left(u_{z}(\xi(t, z))\right)$.

The following result summarizes the sufficient conditions on the diffusion coefficients $\left(\mu^{x}, \sigma^{x}\right)$ and $\left(\mu^{Y}, \sigma^{Y}\right)$, which ensure sufficient regularity for $\mathbf{U}$ to be able to carry out all the calculations. In particular, under these conditions all the results of Section 2.2 are valid.

Theorem 3.1. Let $\delta>0$ and consider a $\mathcal{C}^{2, \delta}$-marginal utility function ${ }^{2} u_{z}$ and $\left\{X_{t}(x)\right\}$ and $\left\{Y_{t}(y)\right\}$ be the solutions of two SDEs with $\mu^{X}$ and $\sigma^{X}$ are in the class $\mathcal{K}_{\text {loc }}^{3, \delta}$ and $\mu^{Y}$ and $\sigma^{Y}$ are in the class $\mathcal{K}_{\text {loc }}^{2, \delta}$. Then the inverse $\{\xi(t, z)\}$ of $\left\{X_{t}(x)\right\}$ is a semimartingale process in $\mathcal{K}_{\text {loc }}^{2, \varepsilon}, \forall \varepsilon<\delta$. Moreover, the random field $Y_{t}\left(u_{z}(\xi(t, z))\right)=U_{z}(t, z)$ is a compatible marginal utility of class $\mathcal{C}^{2, \delta}$.

\footnotetext{
${ }^{2}$ two times differentiable with second derivative is $\delta$-Hölder.
} 
Comment The regularity of SDE's coefficients of the characteristic process implies the existence, the uniqueness, the monotonicity of the solution and that its reverse flow is indeed a semimartingale (which is not always true) of the class $\mathcal{K}_{l o c}^{2, \varepsilon}, \forall \varepsilon<\delta$. The conditions on the SDE's coefficients of $Y$ also imply the existence of a strong monotonic solution of the class $\mathcal{K}_{l o c}^{2, \delta}, \delta>0$. All this, combined with the regularity of $u_{z}$ imply that the marginal utility $\mathbf{U}_{\mathbf{z}}$ is indeed a semimartingale of class $\mathcal{C}^{2}$ (sufficient to apply Itô-Ventzel's formula) and thus its primitive $\mathbf{U}$ is a semimartingale, at least, of class $\mathcal{C}^{3}$ and whose characteristics are the primitive functions of those of $\mathbf{U}_{z}$.

An other consequence of the SDE's regularity for the characteristic process and its adjoint introduced in Theorem 3.1 is their regularity in the neighborhood of 0 and $\infty$. In what follows, $\left\{X_{t}(0)\right\}$ is assumed to be the null process, and $\left\{Y_{t}(\infty)\right\}$ to be equal to $\infty$.

\subsubsection{Limit conditions and intrinsic universe}

An other consequence of the SDE's regularity of the characteristic process and the adjoint process is their regularity near the boundary points 0 and $\infty$ and the martingale property at the limit.

Limit Behavior: Theorem 3.1 implies that the quantity $\frac{x_{t}(x)}{x}$ tends to a limit $\Lambda_{t}^{x}$ a.s, when $x \rightarrow 0$ and that the quantity $\frac{Y_{t}(y)}{y}$ tends to a limit $H_{t}^{Y}$ a.s when $y \rightarrow \infty$. The properties of these limit processes is easier to obtain by writing the differentials in multiplicative form,

$$
d X_{t}(x)=X_{t}(x)\left[\rho_{t}^{x}\left(X_{t}(x)\right) d t+\kappa_{t}^{X}\left(X_{t}(x)\right) \cdot d W_{t}\right], \quad d Y_{t}(y)=Y_{t}(y)\left[\rho_{t}^{Y}\left(Y_{t}(y)\right) d t+\kappa_{t}^{Y}\left(Y_{t}(y)\right) \cdot d W_{t}\right] .
$$

The limit processes $\left\{\Lambda_{t}^{X}\right\}$ and $\left\{H_{t}^{Y}\right\}$ are given by the differential system,

$$
d \Lambda_{t}^{x}=\Lambda_{t}^{x}\left[\rho_{t}^{x}(0) d t+\kappa_{t}^{x}(0) \cdot d W_{t}\right], \quad d H_{t}^{Y}=H_{t}^{Y}\left[\rho_{t}^{Y}(\infty) d t+\kappa_{t}^{Y}(\infty) \cdot d W_{t}\right], \quad \Lambda_{0}^{X}=1, \quad H_{0}^{Y}=1 .
$$

Change of Probability measure The relevant assumption is that $\left.\left\{X_{t}(x) Y_{t}\left(u_{z}(x)\right) / x u_{z}(x)\right)\right\}$ is a martingale, whose limit at $x=0$ is the process $L_{t}=\Lambda_{t}^{X} H_{t}^{Y}, L_{0}=1$. Under regularity assumptions this process is still a martingale.

We often use this positive martingale as the density $\left\{L_{t}\right\}$ w.r. to $\mathbb{P}$ of a new probability measure $\mathbb{Q}$. As explained in Section 2.3, we can only consider the equivalent intrinsic model. In this case, $\Lambda^{x}$ and $H^{Y}$ are normalized to be 1 , so that $\rho_{t}^{x}(0)=0, \quad \kappa_{t}^{X}(0)=0, \quad \rho_{t}^{Y}(\infty)=0, \quad \kappa_{t}^{Y}(\infty)=0$, a.s.

\subsection{Numerical approximation}

Theoretically, we have demonstrated in the previous section how, only from the algebraic compatibility and the orthogonality conditions, we can solve the question of revealed utilities and deduce a lot of information about $\mathbf{U}$. In particular, these two conditions are largely sufficient to characterize the utility dynamics in a semimartingale framework and to associate an optimization problem with it. We are concerned with the second order SPDE satisfied by $\mathbf{U}$, which is, from Proposition 2.5 given by

$$
\left\{\begin{array}{l}
d U(t, z)=\left[\frac{1}{2} U_{z z}(t, z)\left\|\sigma_{t}^{X}(z)\right\|^{2}+z \mu_{t}^{Y}\left(U_{z}(t, z)\right)\right] d t+\gamma^{U}(t, z) d W_{t}, \\
\gamma_{z}^{U}(t, z)=\sigma_{t}^{Y}\left(U_{z}(t, z)\right)-U_{z z}(t, z) \sigma_{t}^{X}(z) .
\end{array}\right.
$$

From an application point of view, one of the most important issues is: which algorithm could be used to numerically approximate $\mathbf{U}$ and what is the of convergence of the numerical scheme?

There are at least two ways to do this.

-The first one is to directly discretize the second order SPDE (3.2), but this is a complex task. We have inevitably to approximate $U_{z}(t, z)$ and $U_{z z}(t, z)$ using a finite differences method for example, which requires the resolution in the full space (or on a grid in $z$ ). This is computationally demanding. In 
addition, it seems really difficult to obtain error estimates in that context, see the detailed discussion in GM18.

- The second method, which is relevant to this work, uses the algebraic compatibility, i.e., the marginal utility is the compound of a SDE's solution $\left\{Y_{t}(y)\right\}$ and the inverse $\xi$ of a first SDE's solution $\left\{X_{t}(x)\right\}$. As forward random field, $\{\xi(t, z)\}$ is solution of a second order SPDE, which does not facilitate the task; the trick is then to simulate the backward flow $\left\{\xi_{s, t}(x), s \leq t\right\}$ ( $t$ is fixed) which is indeed solution of a SDE and in addition $\xi_{0, t}=\xi_{t}$. Using the Backward Brownian motion $\overleftarrow{W}$

$$
\left\{\begin{array}{l}
d \xi_{s, t}(x)=-\left[\mu_{s}^{x}\left(\xi_{s, t}(x)\right)-\sigma_{x}^{x}\left(s, \xi_{s, t}(x)\right) \cdot \sigma_{s}^{x}\left(\xi_{s, t}(x)\right)\right] d s-\sigma_{s}^{x}\left(\xi_{s, t}(x)\right) \cdot d \overleftarrow{W}_{s}, \quad \xi_{t, t}(x)=x \\
\overleftarrow{W}_{s}:=W_{t}-W_{s}, \forall s \leq t
\end{array}\right.
$$

From this, the approximation of $\left\{\xi_{s, t}(x)\right\}$ is made possible simply using a standard Euler scheme, as for $Y$. It remains to be seen whether the compound of the two approximations converges to the compound and at which rate. For well-known issues of adaptation to filtration in numerical probabilities, we make the additional assumption that the coefficients are deterministic functions of time and space. Fortunately, in GM18, the authors have addressed this question in a general framework and one can read directly in [GM18, Theorem 9], the following result under regularities in time of the coefficients ( $\alpha^{x}$ and $\alpha^{Y}$-Hölder).

Theorem 3.2. In addition to Assumptions of Proposition 2.5, suppose that

- the coefficients $\left\{\mu_{t}^{Y}(y), \sigma_{t}^{Y}(y)\right\}$ are deterministic functions of class $\mathcal{K}_{b}^{2, \delta}$ and their derivatives are $\alpha^{Y}$-Hölder in time.

- the coefficients $\left(\mu_{t}^{x}(x)-\sigma_{x}^{x}(t, x) \cdot \sigma_{t}^{x}(x), \sigma_{t}^{x}(x)\right)$ of $\xi_{., t}$ are deterministic functions in the class $\mathcal{K}_{b}^{1, \delta}$ and their derivatives are $\alpha^{x}$-Hölder in time.

Denote by $Y_{0, .}^{N}$ the Euler approximation $Y$, with time step $T / N$, and by $\xi_{., t}^{N}$ the Euler approximation of the inverse flow $\xi_{., t}$ of $X$, with time step $T / N$.

Then, for any concave function u with Lipschitz marginal utility $u_{z}$, the compound Euler scheme $Y^{N}\left(u_{z}\left(\xi^{N}\right)\right)$ converges to $U_{z}(.,$.$) (the marginal utility of U$, solution to the SPDE (3.2) in any $\mathbf{L}_{p}$-norm, at the order $\beta:=\min \left(\alpha^{x}, \alpha^{Y}, \frac{1}{2}\right)$ w.r.t. $N:$ For any $p>0$ and any $t \in[0, T]$,

$$
\left\|Y_{t}^{N}\left(u_{z}\left(\xi_{0, t}^{N}(z)\right)\right)-U_{z}(t, z)\right\|_{\mathbf{L}_{p}}=O\left(N^{-\beta}\right)
$$

Now we have at hand an accurate approximation of $U_{z}(t, z)$ using two Euler schemes (with computational cost equal to $2 N$ ), we can easily retrieve $U(t, z)$ by standard numerical integration, using a known value of $U\left(t, z_{0}\right)$ for some $z_{0}$ (here, we have $U(t, 0)=0$ ).

\subsection{Strong orthogonality and financial market}

We continue our investigations on the bi-revealed utilities when the strong suborthogonality in $\mathcal{A} Z$ conditions is replaced by the strong orthogonality of the processes $\left\{x_{t}(x), Y_{t}(y)\right\}$, that is

$$
\left\{X_{t}(x) Y_{t}(y)\right\} \text { is martingale for any }(x, y) \text {. }
$$

The system $(u, X, Y)$ is a particular case of the $\mathcal{A} Z$-system, satisfying the limit conditions under Itô regularity's assumptions. We start with the intrinsic model, which makes intuition easy to develop.

\subsubsection{The intrinsic martingale framework}

Let us assume the intrinsic condition, $\left(X_{t}^{\text {int }}(x) / x \rightarrow 1, Y_{t}^{\text {int }}(y) / y \rightarrow 1\right)$.

The main difference with the $\mathcal{A} Z$-condition is that many processes become martingales. We start with a characterization of $Y^{\text {int }}$ - strongly orthogonal processes in the intrinsic market. 
Proposition 3.2. Under the strong orthogonality assumption: $\left\{X_{t}^{\text {int }}(x) Y_{t}^{\text {int }}(y)\right\}$ are $\mathbb{Q}^{\text {int }}$-martingales, - The processes $\left\{X_{t}^{\mathrm{int}}(x)\right\}$ and $\left\{Y_{t}^{\mathrm{int}}(y)\right\}$ are $\mathbb{Q}^{\mathrm{int}}$-martingales.

More generally, any positive semimartingale $\left\{Z_{t}^{\text {int }}\right\}$, strongly orthogonal to $\left\{Y_{t}^{\text {int }}(y)\right\}$ verifies,

- the process $\left\{Z_{t}^{\text {int }}\right\}$ itself is a $\mathbb{Q}^{\text {int }}$-martingale

- the strong orthogonality with $Y_{t}^{\mathrm{int}}(y)$ is equivalent to a volatility property:

"the intrinsic volatility vectors of $\left\{Z_{t}^{\text {int }}\right\}$ and $\left\{Y_{t}^{\text {int }}(y)\right\}$ are orthogonal for any $y "$.

Obviously, this properties can be expressed in terms of the (non intrinsic) original market, based on the processes $\left\{\Lambda_{t}^{X}, H_{t}^{Y}\right\}$, defining the class of standard processes $\left\{X_{t}(x)=X_{t}^{\text {int }}(x) \Lambda_{t}^{x}\right\}$ and $\left\{Y_{t}(y)=\right.$ $\left.Y^{\mathrm{int}}(y) H_{t}^{Y}\right\}$. The "intrinsic" property is that $\left\{L_{t}^{\mathrm{int}}=\Lambda_{t}^{x} H_{t}^{Y}\right\}$ is a $\mathbb{P}$-martingale.

To minimize calculations, we exploit the fact that the volatility of a semimartingale is invariant by change of probability measure.

Volatility Calculations: (i) Let us denote by $\left(\kappa_{t}^{x}(x), \kappa_{t}^{Y}(y)\right)$ the volatility vectors of $\left\{X_{t}(x), Y_{t}(y)\right\}$ and by $\left(\kappa_{t}^{Z}, \kappa_{t}^{\Lambda}, \kappa_{t}^{H}\right)$ the volatility vectors of $\left\{Z_{t}, \Lambda_{t}, H_{t}^{Y}\right\}$ with $\left(\kappa_{t}^{\Lambda}=\kappa_{t}^{X}(0), \kappa_{t}^{H}=\kappa_{t}^{Y}(\infty)\right.$.

(ii) The intrinsic volatilities are deduced of the definition of intrinsic processes, given that the volatility of a product is the sum of the volatility.

- The intrinsic volatility of $\left\{X_{t}^{\text {int }}(x)=X_{t}(x) / \Lambda_{t}^{X}\right\}$ is $\kappa_{t}^{X \text {,int }}(x)=\kappa_{t}^{X}(x)-\kappa_{t}^{\Lambda}$.

- The intrinsic volatility of $\left\{Y_{t}^{\text {int }}(y)\right\}=\left\{Y_{t}(y) / H_{t}^{Y}\right\}$ is $\kappa_{t}^{Y, \text { int }}(y)=\kappa_{t}^{Y}(y)-\kappa_{t}^{H}$.

- The intrinsic volatility of $\left\{Z_{t}^{\text {int }}\right\}=\left\{Z_{t} / \Lambda_{t}^{X}\right\}$ is $\kappa_{t}^{Z \text {,int }}=\kappa_{t}^{Z}-\kappa_{t}^{\Lambda}$.

The intrinsic strong orthogonality criterium of Proposition 3.2 is very efficient to pass to the strong orthogonality in the standard framework, using the linear correspondance $\left\{Z_{t}=Z_{t}^{\mathrm{int}} \Lambda_{t}^{x}\right\}$.

Theorem 3.3 (From one universe to the other).

(i) Strong orthogonality equivalence: $\left\{Z_{t}^{\text {int }}\right\}$ is strongly $\mathbb{Q}^{\text {int }}$-orthogonal to $\left\{Y_{t}^{\text {int }}(y)\right\}$ if and only if $\left\{Z_{t}\right\}$ is strongly $\mathbb{P}$-orthogonal to $\left\{Y_{t}(y)\right\}$

(ii) A equivalent condition is that $\left\{Z_{t} H_{t}^{Y}\right\}$ is a $\mathbb{P}$-martingale and

(Volatility condition) $\left\langle\kappa_{t}^{Z}-\kappa_{t}^{\Lambda}, \kappa_{t}^{Y}(y)-\kappa_{t}^{H}\right\rangle=0$, a.s. $d t \otimes d \mathbb{P}$,

(iii) Any $Y$-strong orthogonal process, $\left\{Z_{t}\right\}$ is controlled by its volatility $\kappa_{t}^{Z}$,

$$
d Z_{t}=Z_{t}\left[-\rho_{t}^{H} d t+\kappa_{t}^{Z} \cdot\left(d W_{t}-\kappa_{t}^{H} d t\right)\right] \quad \text { under the constraint }\left\langle\kappa_{t}^{Z}-\kappa_{t}^{\Lambda}, \kappa_{t}^{Y}(y)-\kappa_{t}^{H}\right\rangle=0, \forall y .
$$

(iv) The characteristic process evolves as

$$
\left\{\begin{array}{l}
d X_{t}(x)=X_{t}(x)\left[-\rho_{t}^{H} d t+\kappa_{t}^{X}\left(X_{t}(x)\right) \cdot\left(d W_{t}-\kappa_{t}^{H} d t\right)\right] \\
\left\langle\kappa_{t}^{x}(x)-\kappa_{t}^{\Lambda}, \kappa_{t}^{Y}(y)-\kappa_{t}^{H}\right\rangle=0, \quad \forall(y, x) .
\end{array}\right.
$$

(v) The revealed utility $U\left(t, Z_{t}\right)$ is a supermartingale and a martingale when $Z_{t}=X_{t}(x)$.

\subsubsection{Links with financial market}

Equation 3.5 remind us a standard framework of financial market, where the underlying asset, here the characteristic process $\left(X_{t}(x)\right)$ is strongly orthogonal to an adjoint processes $\left\{Y_{t}(y)\right\}$, often called "pricing kernel", because of the orthogonality relation $\mathbb{E}\left(X_{t}(x) Y_{t}(y) / y\right)=x$. Moreover, in finance, the volatility vector $\kappa_{t}^{Y}(y)$ is assumed to be orthogonal to the vector $\kappa_{t}^{X}(x)$, or only to the vector $\bar{\kappa}_{t}^{X}=\kappa_{t}^{X}(0)$, from the strong orthogonality condition.

The optional process $\left(-\rho_{t}^{H}=r_{t}\right)$ is interpreted as the short rate and $\left(-\kappa_{t}^{H}\right)$ as the risk premium of the market. 
The originality of our point of view is that the whole construction of the market is based on two processes, strongly orthogonal and on a initial utility. Nevertheless, the revealed utility is effectively the value function of a portfolio optimization problem. The intrinsic universe can be intrepreted as a risk-neutral financial market.

This presentation of the portfolio optimization problem does not make any reference to the notion of arbitrage and self-financing, but only to the orthogonality with an "optimal" state pricing process.

\subsection{Application to aggregation problem}

The problem of the aggregation of preferences is a standard problem in convex analysis, especially in economy, where the problem is very intuitive and used as a reference framework.

Consider a group of agents classified into classes (indexed by $\theta$ ) having the same preferences $u^{\theta}$ for a wealth amount $x^{\theta}$. The weight of the different classes is quantified by a finite positive measure $\mu(d \theta)$ on a metric space $\Theta$. The global wealth $z$ of the economy is $\int x^{\theta} \mu(d \theta)=z$. So, $z$ is shared between the different agents through the family of functions $z \rightarrow x^{\theta}(z)$. To guarantee the monotonicity of the various aggregated quantities, the functions $z \rightarrow x^{\theta}(z)$ are assumed to be increasing and differentiable, with range $(0, \infty)$. The simplest example is the linear case, $x^{\theta}(z)=\alpha^{\theta} z$ with $\int \alpha^{\theta} \mu(d \theta)=1$ (as in [EKHM17]).

The next step is to define the utility of a "representative agent" of the aggregated economy. As usual in convex analysis, the aggregation concerns the marginal utilities and not the utilities themselves. So, under the assumption that $u_{z}^{\theta}\left(x^{\theta}(z)\right)$ is $z$-integrable in $z=0$, the marginal utility (and then the utility) of the aggregated problem is defined by:

$$
u_{z}^{(\mu)}(z)=\int_{\Theta} u_{z}^{\theta}\left(x^{\theta}(z)\right) \mu(d \theta), \quad z=\int_{\Theta} x^{\theta}(z) \mu(d \theta)
$$

By analogy, put $\bar{y}^{\theta}\left(u_{z}^{(\mu)}(z)\right)=u_{z}^{\theta}\left(x^{\theta}(z)\right)$, then

$$
\tilde{u}_{y}^{(\mu)}(y)=\int_{\Theta} \tilde{u}_{y}^{\theta}\left(\bar{y}^{\theta}(y)\right) \mu(d \theta), \quad y=\int_{\Theta} \bar{y}^{\theta}(y) \mu(d \theta) .
$$

Our aim is to characterize a representative agent of the aggregated population and his representative preference. In the dynamic framework, the agents are classified by their forward dynamic utility $\mathbf{U}^{\theta}$ and their "characteristic" wealth $\left(X^{\theta}, x\right)$, their initial utility $u^{\theta}$ as well as by their adjoint process $Y_{t}^{\theta}\left(u_{z}^{\theta}(x)\right)=$ $U_{z}^{\theta}\left(t, X_{t}^{\theta}(x)\right)$. The initial properties on wealth sharing and aggregating initial marginal utilities can be generalized without modification to the dynamic framework, into

$$
\left\{\begin{array}{l}
x_{t}^{(\mu)}(z)=\int X_{t}^{\theta}\left(x^{\theta}(z)\right) \mu(d \theta) \\
U_{z}^{(\mu)}\left(t, X_{t}^{(\mu)}(z)\right)=\int_{\Theta} U_{z}^{\theta}\left(t, X_{t}^{\theta}\left(x^{\theta}(z)\right)\right) \mu(d \theta) \\
Y_{t}^{(\mu)}\left(u_{z}^{(\mu)}(x)\right):=\int_{\Theta} Y_{t}^{\theta}\left(u_{z}^{\theta}\left(x^{\theta}(z)\right)\right) \mu(d \theta)=\int Y_{t}^{\theta}\left(\bar{y}^{\theta}\left(u_{z}^{(\mu)}(z)\right)\right) \mu(d \theta) .
\end{array}\right.
$$

The last question is to specify under which conditions the aggregation of revealed utilities is still a revealed utility. The dynamic utility $\mathbf{U}^{(\mu)}$, which is compatible with $\left(X^{(\mu)}, \mathbf{Y}^{(\mu)}, u^{(\mu)}\right)$, is expected to satisfy that $\left\{U\left(t, X_{t}(x)\right)\right\}$ is a martingale. We have seen that a sufficient condition is the strong orthogonality of the processes $\left(X^{(\mu)}, \mathbf{Y}^{(\mu)}\right)$ which will be obtained for instance from the strong orthogonality of the family $\left(X^{\theta}, \mathbf{Y}^{\theta^{\prime}}\right)$.

Proposition 3.3. Consider the characteristic processes of the aggregated economy $\left(X^{(\mu)}, \mathbf{Y}^{(\mu)}, u\right)$ defined by equations 3.9, 3.6, 3.10. Assume that : 
for any $\left(\theta, \theta^{\prime}, x, y\right), X_{t}^{\theta}(x) Y_{t}^{\theta^{\prime}}(y)$ is a martingale.

Then, the processes $\left\{X_{t}^{(\mu)}(x)\right\}$ and $\left\{Y_{t}^{(\mu)}(y)\right\}$ are strongly orthogonal and the utility $\mathbf{U}^{(\mu)}$ defined in (3.6) is a revealed utility with characteristic process $\left\{X_{t}^{(\mu)}(x)\right\}$.

Proof. From previous results, it is sufficient to show that $\left(X^{(\mu)}, \mathbf{Y}^{(\mu)}\right)$ are strongly orthogonal, or equivalently that $\left\{X_{t}^{(\mu)}(x) Y_{t}^{(\mu)}(y)\right\}$ is a martingale. By (positive) Fubini's Theorem, and the definition of $X^{(\mu)}$, and $\mathbf{Y}^{(\mu)}$,

$$
X_{t}^{(\mu)}(x) Y_{t}^{(\mu)}(y)=\iint \mu(d \theta) \mu\left(d \theta^{\prime}\right) X_{t}^{\theta}\left(x^{\theta}(x)\right) Y_{t}^{\theta^{\prime}}\left(y^{\theta^{\prime}}(y)\right) .
$$

The martingale property of $\left\{X_{t}^{\theta}(z) Y_{t}^{\theta^{\prime}}(y)\right\}$ is extended to the product $\left\{X_{t}^{(\mu)}(x) Y_{t}^{(\mu)}(y)\right\}$, once again thanks to positive Fubini's theorem.

Links with the Pareto wealth allocation We come back to the definition of the initial marginal utility from the wealth sharing functions $\left\{x^{\theta}(x)\right\}$. The question of the "optimal" choice of these functions is related with the following optimization problem, known as the Pareto allocation problem: "Find the best allocation $\left\{x^{\theta}(x)\right\}$ such that $\int_{\Theta} x^{\theta}(x) \mu(d \theta)=x$, maximizing the sup-convolution criterium $\sup \int_{\Theta} u^{\theta}\left(x^{\theta}(x)\right) \mu(d \theta)$ " see Proposition 4.2 . An optimal solution $\left\{x^{\theta, *}(x)\right\}$, (if there exists), must satisfy the first order condition on the marginal rate of substitution, $\left(\forall x, \forall \theta, u_{z}^{\theta}\left(x^{\theta, *}(x)\right)=u_{z}(x)\right)$. This optimal choice is Pareto optimal in the sense that the wealth is allocated in the most efficient manner, but this choice does not imply equality or fairness. The extension to dynamic processes and forward utility is discussed in Section 5.2 of [EKM13].

\section{Revealed non-concave preferences and economic equilibrium}

The first motivation of this section is to extend the previous construction to a regular SDE's system with coupled coefficients, in order to solve equilibrium problems. The paper by He and Leland [HL93] on the necessary conditions for equilibrium has been a rich source of inspiration, and we have adapted their ideas in our context.

\subsection{Partially coupled SDEs system}

We adopt a similar presentation than in Sections 2.2 and 3 and still assume the strong suborthogonality condition of Theorem 2.2 but we suppose now that the pair $(X, Y)$ is a regular solution of a partially coupled differential system, whose first component $X$ is assumed to be autonomous.

$$
\operatorname{Syst}(I I)\left\{\begin{array}{lc}
d X_{t}(x)=\mu_{t}^{x}\left(X_{t}(x)\right) d t+\sigma_{t}^{x}\left(X_{t}(x)\right) d W_{t}, & X_{0}(x)=x, \\
d Y_{t}(x, y)=\mu_{t}^{Y}\left(X_{t}(x), Y_{t}(x, y)\right) d t+\sigma_{t}^{Y}\left(X_{t}(x), Y_{t}(x, y)\right) d W_{t}, & Y_{0}(x, y)=y .
\end{array}\right.
$$

Regularity assumptions are made on the coefficients of the system to guarantee existence, uniqueness, regularity and positivity of the solution $\left(X_{t}(x), Y_{t}(x, y)\right)$ of the SDE's system. The multiplicative form of the coefficients is for $X,\left(\mu_{t}^{x}(x)=x \rho_{t}^{x}(x), \sigma_{t}^{x}(x)=x \kappa_{t}^{x}(x)\right)$ and for $Y\left(\mu_{t}^{Y}(x, y)=y \rho_{t}^{Y}(x, y), \sigma_{t}^{Y}(x, y)=\right.$ $\left.y \kappa_{t}^{Y}(x, y)\right)$.

Under the regularity assumptions, $x \rightarrow X_{t}(x)$ is increasing, and the process $y \rightarrow Y_{t}(x, y)$ is increasing for a given $x$. But the function $x \rightarrow Y_{t}(x, y)$ is more complex to analyze, and is not increasing in general. In [EKM13] and Proposition 3.1. we have studied the limit behavior of $X_{t}(x) ; X_{t}(x) \rightarrow 0$ when $x \rightarrow 0$ and to $+\infty$ when $x \rightarrow+\infty$. Moreover, the uniform Lipschitz assumption on $\mu^{Y}$ implies that if $(y \rightarrow \infty, x \rightarrow 0)$ then $\lim \rho_{t}^{Y}(x, y) \rightarrow+\infty$. We also need the more precise result that $x Y_{t}(x, y) \rightarrow 0$ if 
$x \rightarrow 0, y \rightarrow \infty$, and $x y \rightarrow 0$. Such result can be in general deduced from the regularity of the coefficients (or taken as an assumption).

\subsubsection{SPDE of non concave preferences}

Let us consider a deterministic (decreasing) function $v$, of class $\mathcal{C}^{2}$ on $(0, \infty)$, with limit $\infty$ at 0 , and 0 at $\infty$. We are concerned with the random field $\widehat{Y}(t, x)=Y_{t}(x, v(x)), x>0$, whose differential characteristics are $\widehat{\beta}^{Y}(t, x)=\mu_{t}^{Y}\left(X_{t}(x), \widehat{Y}(t, x)\right)$, and $\widehat{\gamma}^{Y}(t, x)=\sigma_{t}^{Y}\left(X_{t}(x), \widehat{Y}(t, x)\right)$. This random field is no longer decreasing in $x$, but sufficiently regular to apply Itô-Ventzel's formula, and its limit when $x \rightarrow 0$ is well-understood.

General SPDE: As previously, we denote by $\{\xi(t, z)\}$ the regular inverse of the monotonic process $\left\{X_{t}(x)\right\}$. For any $\mathcal{C}^{2}$-function $v$, we introduce the compound random field $G(t, z)=Y_{t}(\xi(t, z), v(\xi(t, z)))$, also denoted $\widehat{Y}(t, \xi(t, z))$. To deduce the dynamics of the primitive $J$ of $G$, we can also apply the result of Proposition 2.5 since in its proof the latter does not require concavity. The calculations are identical, except that we have to replace $\gamma^{Y}\left(t, u_{z}(z)\right)$ by $\sigma_{t}^{Y}\left(X_{t}(z), Y_{t}(z, v(z))\right.$ and $\beta^{Y}\left(t, u_{z}(z)\right)$ by $\mu_{t}^{Y}\left(X_{t}(z), Y_{t}(z, v(z))\right.$. The main difference is that $G$ still a positive regular random field on $(0, \infty)$, but in general not decreasing (not a marginal utility). Assuming the $v$-orthogonality condition: $\left\{X_{t}(x) Y_{t}(x, v(x))\right\}, \forall x$, we obtain the same SPDE than in the decoupled case for the primitive $J$ of $G$.

Theorem 4.1. Let $J(t, z)$ (with $J(t, 0)=0$ ) be the primitive preferences of $G(t, z)=Y_{t}(\xi(t, z), v(\xi(t, z))$ ). Assume $X$ and $Y$ satisfy the strong sub-orthogonality condition of Theorem 2.2. then

(i) From Proposition 2.5.

$$
\left\{\begin{array}{l}
d J(t, z)=\left[z \mu_{t}^{Y}\left(z, J_{z}(t, z)\right)+\frac{1}{2}\left\|\sigma_{t}^{X}(z)\right\|^{2} J_{z z}(t, z)\right] d t+\gamma^{J}(t, z) d W_{t} \\
\gamma_{z}^{J}(t, z)=\sigma_{t}^{Y}\left(z, J_{z}(t, z)\right)-J_{z z}(t, z) \sigma_{t}^{X}(z) .
\end{array}\right.
$$

Nevertheless, the concavity is not guaranteed in general.

(ii) As before, the process $J\left(t, X_{t}(x)\right)$ is a martingale, but in absence of concavity of $J$, it is not an optimal choice.

Remark . (i) Since the J-SPDE is similar of the SPDE of the decoupled case, equation 2.6 holds,

$$
\begin{aligned}
d J\left(t, Z_{t}\right) & =\frac{1}{2} J_{z z}\left(t, Z_{t}\right)\left\|\sigma_{t}^{X}\left(Z_{t}\right)-\psi_{t}^{Z}\right\|^{2} d t+\left\langle\gamma^{J}\left(t, Z_{t}\right)+J_{z}\left(t, Z_{t}\right) \psi_{t}^{Z}, d W_{t}\right\rangle \\
& +\left[Z_{t} \mu_{t}^{Y}\left(Z_{t}, J_{z}\left(t, Z_{t}\right)\right)+J_{z}\left(t, Z_{t}\right) \phi_{t}^{Z}+\left\langle\sigma_{t}^{Y}\left(Z_{t}, J_{z}\left(t, Z_{t}\right)\right), \psi_{t}^{Z}\right\rangle\right] d t .
\end{aligned}
$$

By the orthogonality condition, the martingale property of $Z=X$ holds, but the optimality is lost since the sign of $J_{z z}\left(t, Z_{t}\right)$ is not negative.

(ii) If $\widehat{Y}(t, x)=Y_{t}(x, v(x))$ is monotonous with respect to $x$, then $J(t, z)$ is a compatible utility $U(t, z)$.

Comment: To summarize, in Sections 2.2 and 2.3 , we have shown that for any decoupled system satisfying the hypothesis of Theorem 2.2, and for any concave $\mathcal{C}^{3}$-function $u$, the construction by composition of two regular random fields yields to a revealed utility $\mathbf{U}$, solution of the SPDE given in Proposition 2.5. with optimality of the characteristic process $Z=X$ and its orthogonal process $Y$. When the system is partially coupled and hypothesis of Proposition 2.2 holds, the same process yields for $J$ to the same SPDE [4.3), and the martingale property for $J\left(t, x_{t}(x)\right)$, but many differences appear with the loss of the decreasing property of $x \rightarrow Y_{t}(x, v(x))$, and then of the concavity of $J$. To find a concave solution, the initial condition $v=u_{z}$ cannot be any, as well as the random multiplicative coefficients $\kappa_{t}^{X}(x), \kappa_{t}^{Y}(x, y)$, the other coefficients being determined by the orthogonality conditions (up to stochastic initial conditions). The problem is to find motivated conditions yielding to the existence of a triplet $(u, \mathcal{X}, Y)$ for which a revealed utility can exist. 


\subsubsection{Discussion on the orthogonality condition}

As explained at the beginning of this section, we are concerned with the application of our results to solve the economic equilibrium problem as introduced in the work of He and Leland [HL93]. For this, we need to clarify a little more the assumptions satisfied in the latter work.

ORTHOGONALITY CONDITIONS: These conditions are, for the rest of the paper, of two types: orthogonality condition of Theorem 2.2 in particular the coordinates $X(x)$ and $\widehat{Y}(t, x)=Y_{t}(x, v(x))$ are orthogonal and the $v$-orthogonality of $\widehat{Y}(t, x)$ and the derivative $X_{x}(t, x)$. This leads to $\left\{X_{t}(x) Y_{t}(x, v(x)), x>0\right\}$ and $\left\{Y_{t}(x, v(x)) X_{x}(t, x), x>0\right\}$ are assumed to be martingales.

Remark . It is important to note that the second hypothesis is always satisfied, because it is simply the consequence of the regularity of $X: U\left(t, X_{t}(x)\right.$ is a martingale is equivalent, deriving in $x$, to $X_{x}(t, x) U_{z}\left(t, X_{t}(x)\right)$ is a martingale. It is therefore not a veritable assumption.

The first martingale condition of the coordinates is equivalent to the following constraints on the coefficients

$$
(I)\left\{\begin{array}{c}
z \mu_{t}^{Y}(z, G(t, z))+G(t, z) \mu_{t}^{x}(z)+\left\langle\sigma_{t}^{x}(z), \sigma_{t}^{Y}(z, G(t, z))\right\rangle=0 \\
\text { or } \rho_{t}^{Y}(z, G(t, z))+\rho_{t}^{x}(z)+\left\langle\kappa_{t}^{x}(z), \kappa_{t}^{Y}(z, G(t, z))\right\rangle=0 .
\end{array}\right.
$$

The second assumption concerns the $v$-orthogonality of $\left\{Y_{t}(x, v(x))\right\}$ and the derivative $\left\{X_{x}(t, x)\right\}$ for any $x>0$. An equivalent formulation, where $y$ is replaced by $G(t, z)$, is

$$
(v I I)\left\{\begin{array}{l}
\mu_{t}^{Y}(z, G(t, z))+G(t, z) \mu_{z}^{x}(t, z)+\left\langle\sigma_{z}^{x}(t, z) \sigma_{t}^{Y}(z, G(t, z))=0,\right. \\
\operatorname{or} \rho_{z}^{x}(t, z)+\left\langle\kappa_{z}^{x}(t, z), \kappa_{t}^{Y}(z, G(t, z))\right\rangle=0 .
\end{array}\right.
$$

The main difficulty is the dependence of the constraint in $v$ and more generally in $G$.

\subsection{Random economic equilibrium and their time-finite variation decreasing preferences}

Our aim is to introduce additional assumptions to simplify the problem of existence of a concave solution to the $J$-SPDE 4.3 . The new setting is motivated by the standard Markovian point of view in economy, in particular by the framework of He and Leland [HL93] on the economic equilibrium. The $J$ function is assumed to be concave and deterministic, with finite variation in time, (decreasing for concave function). In random environment (our framework) this notion is extended into preferences with finite variation in time, first studied by Musiela and Zariphopoulou [MZ10a] and Berrier \& al [BRT09] in a more restrictive setting. Moreover, guided by economic considerations in absence of consumption, the short rate $r_{t}$ is assumed to be exogenous but stochastic, and equal (by assumption) to the opposite of the relative drift coefficient $\rho_{t}^{Y}(x, y)=-r_{t}$ of the $Y$-process.

Besides the fact that we completely solve this equilibrium problem, which to our knowledge remains an open problem until now, one of the important points to retain in this section is that the characteristic process $X$, its adjoint $Y$ and the initial utility $u$ are very constrained, therefore the problem does not admits a solution for any triplet $(u, X, Y)$.

\subsubsection{A random economic equilibrium setting}

We define a random economic equilibrium setting as an universe generated by a partially coupled strongly orthogonal system $\left(X_{t}(x), Y_{t}^{e}(x, y)\right)$ (e for equilibrium), as before the relative drift $\rho_{t}^{Y}(x, y)$ of $Y^{e}$, is equal to $-r_{t}$. In addition, we only consider dynamic preferences with initial condition $u$, such that 
$\left(X_{x}(t, x) Y_{t}\left(x, u_{z}(x)\right)\right)$ are orthogonal, that are of finite variation in time, meaning that the $G$-diffusion random field $\gamma_{z}(t, z)=0$. In the following lemma, we explain the role of these two different assumptions.

Proposition 4.1. Under the two orthogonality constraints $(I)$ and $(v I I)$,

i-a) If $\rho_{t}^{Y}(x, y)=-r_{t}$, there exists a process $\eta_{t}^{e}$ such that $\left(\kappa_{t}^{Y}(z, G)+\eta_{t}^{e}\right)$ is orthogonal to $\kappa_{t}^{x}(z)$.

i-b) If $G$ is of finite variation in time, then $G(t, z) \kappa_{t}^{Y}(z, G)=z G_{z}(t, z) \kappa_{t}^{x}(z)$ so that the vectors $\kappa_{t}^{Y}(z, G)$ and $\kappa_{t}^{x}(z)$ are collinear for any $z$.

i-c) If the two properties hold together, only the volatility $\kappa^{X}$ of $X$ is free and $\forall z>0$,

$$
\kappa_{t}^{Y}(z, G)=-\eta_{t}^{e}, \quad \rho_{t}^{x}(z)=r_{t}+\left\langle\kappa_{t}^{x}(z), \eta_{t}^{e}\right\rangle, \quad \rho_{t}^{Y}(z, G)=-r_{t}, d t \otimes \mathbb{P} \text {, a.s. }
$$

(ii) The equilibrium setting is similar to the one of a financial market, described in Section 3.3.2:

a) The adjoint process $Y^{e}=y \bar{Y}_{t}^{e}$ is independent of $x$ and linear with respect to $y$,

$$
Y_{t}(x, y)=y \exp \left(-\int_{0}^{t}\left(r_{s}+\frac{1}{2}\left\|\eta_{s}^{e}\right\|^{2}\right) d s-\int_{0}^{t} \eta_{s}^{e} \cdot d W_{s}\right)=y \bar{Y}_{t}^{e} .
$$

b) $\left(X_{t}(x), Y_{t}^{e}(y)\right)$ defines a decoupled strongly orthogonal system. Consequently, from results of Section 2.2. for any initial regular utility, the random field $\mathbf{J}$ is bi-revealed utility and is the value function of some portfolio optimization problem. The linear adjoint process is optimal for the conjugate utility $\widetilde{\mathbf{J}}$.

Proof. (i) Taking the difference between the derivative of $(I)$ (w.r.t. $z$ ) and $(v I I)$ leads to the identity $\partial_{z}\left(\rho_{t}^{Y}(z, G(t, z))\right)+\left\langle\partial_{z}\left(\kappa_{t}^{Y}(z, G(t, z)), \kappa_{t}^{X}(z)\right)\right\rangle=0, \forall z$. If $\rho_{t}^{Y}(z, G(t, z))$ is independent of $z$, then $\left\langle\kappa_{t}^{X}(z), \partial_{z}\left(\kappa_{t}^{Y}(z, G(t, z))\right\rangle=0, \forall z\right.$. That is the vector $\partial_{z}\left(\kappa_{t}^{Y}(z, G(t, z))\right.$ is orthogonal to $\kappa_{t}^{X}(z)$ for every $z$. So there exists a random field $\eta_{t}^{e}$ such that $\left\langle\kappa_{t}^{Y}(z, G)+\eta_{t}^{e}, \kappa_{t}^{X}(z)\right\rangle=0$.

If $J$ is with finite variation, then its volatility vector is null almost surely for every $z, \gamma^{G}(t, z)=$ $\sigma_{t}^{Y}(z, G(t, z))-G_{z}(t, z) \sigma_{t}^{X}(z)=0$, that is $G(t, z) \kappa_{t}^{Y}(z, G(t, z))=z G_{z}(t, z) \kappa_{t}^{x}(z)$ and the vectors $\kappa_{t}^{Y}(z, G)$ and $\kappa_{t}^{x}(z)$ are collinear. If the two properties hold together, $\kappa^{Y}$ is collinear to $\kappa^{x}$ which implies that $\kappa_{t}^{Y}(z, G(t, z))=-\eta_{t}^{e}$ is also independent of $z$.

(ii) The representation of $Y_{t}^{e}(x, y)$ is then standard, and by construction the processes $X_{t}(x)$ and $\bar{Y}^{e}$ are strongly orthogonal. All the other properties are shown in Section 2.2, since strong orthogonality implies the sub orthogonality.

Then the problem is now to find a pair $\left(u_{z}, x_{t}(x)\right)$ such that the revealed utility with a linear adjoint process is decreasing in time. Clearly, it is very natural to consider first the dual problem whose optimal solution $y \bar{Y}_{t}^{e}$ is known to be linear.

\subsection{Characterization of the equilibrium preferences}

With these different assumptions, at the equilibrium, the market parameters $\left(r_{t}, \eta_{t}^{e}\right)$ are exogenous. Then, the forward SPDE given in Proposition 2.4 becomes, since $\gamma^{\widetilde{J}}=\gamma^{J}=0$,

$$
\partial_{t} \widetilde{J}(t, y)+\frac{1}{2} y^{2}\left\|\eta_{t}^{e}\right\|^{2} \widetilde{J}_{y y}(t, y)-y r_{t} \widetilde{J}_{y}(t, y)=0
$$

The question is to characterize all solutions of this forward PDE considered $\omega$ by $\omega$.

\subsubsection{Characterization of the forward solutions of the duale SPDEs 4.12}

It is easier to find a solution to 4.12 in the family of time-separable conjugate utilities, $\widetilde{J}(t, y)=j(y) H_{t}$ since dual power utilities are obviously good candidates given by

$$
\widetilde{J}^{(\beta)}(t, y)=\tilde{H}_{t}^{(\beta)} \frac{y^{1-\beta}}{\beta-1}, \quad \tilde{H}_{t}^{(\beta)}=\exp \left(-(\beta-1) \int_{0}^{t}\left(r_{s}+\frac{1}{2} \beta\left\|\eta_{s}^{e}\right\|^{2}\right) d s\right), \quad \beta>1
$$


But they are not the only ones. We provide a complete characterization of all positive time-decreasing solutions, with the help of the Widder theorem characterizing the positive space-time harmonic functions of the Brownian motion. This result was first used by Musieal and Zariphopoulou [MZ10a], Berrier \& al [BRT09, in the context of dynamic utilities.

Theorem 4.2 (Widder 1963). A function $\Psi:(0, \infty) \times \mathbb{R} \rightarrow \mathbb{R}$ is a positive standard solution to the heat equation, $\Psi_{t}(t, z)+\frac{1}{2} \Psi_{z z}(t, z)=0$, if and only if it can be represented as

$$
\Psi(t, z)=\int_{\mathbb{R}} e^{\beta z-\frac{1}{2} \beta^{2} t} m(d \beta),
$$

where $m$ is a Borel measure such that the above integral is finite for all $(t, z) \in(0, \infty) \times \mathbb{R}$.

The "revealed conjugate utility" version of this theorem is the following,

Theorem 4.3. A regular function $\widetilde{J}$ is a revealed conjugate utility if and only if there exists a positive Borel measure $\mu(d \beta)$ on $(1, \infty)$ such that $\tilde{u}(y)=\int_{1}^{\infty} \frac{y^{1-\beta}}{\beta-1} \mu(d \beta)\langle\infty$, and that

$$
\widetilde{J}^{(\mu)}(t, y)=\int_{1}^{\infty} \widetilde{J}^{(\beta)}(t, y) \mu(d \beta)=\int_{1}^{\infty} \tilde{H}_{t}^{\beta} \frac{y^{1-\beta}}{\beta-1} \mu(d \beta),
$$

where $\tilde{H}_{t}^{\beta}=\exp \left(-(\beta-1) \int_{0}^{t}\left(r_{s}+\frac{1}{2} \beta\left\|\eta_{s}^{e}\right\|^{2}\right) d s\right)$ is decreasing in time.

$\widetilde{J}^{(\mu)}$ is a solution of the PDE 4.12) whose initial conjugate utility is necessarily the aggregation of conjugate power utilities. Any revealed conjugate utility is the aggregated dual utility of a family of heterogenous risk averse agents, with the same linear pricing kernel given by 4.10.

Proof. The proof is based on the representation of the geometric Brownian motion as a time-dependent function of changed time Brownian motion. In addition of the square integrability of $\eta^{e}$, assume $\left\|\eta_{t}^{e}\right\|>0$ for every $t$. After a standard change of time driven by the inverse of the increasing process $A_{t}=\int_{0}^{t}\left\|\eta_{s}^{e}\right\| d s$, we can assume for simplicity that $\left\|\eta_{t}^{e}\right\| \equiv 1$ for any $t$, and drop out $\eta_{t}^{e}$ in the PDE 4.12 and replacing $r_{t}$ by $\tilde{r}_{t}=r_{t} /\left\|\eta_{t}^{e}\right\|^{2}$. The process $\left\{\tilde{Y}_{t}^{e}(y)\right\}$ is a time depending function of the Brownian motion $\left\{\tilde{W}_{t}=-W_{t}\right\}$, $\tilde{Y}_{t}^{e}\left(e^{z}\right)=\exp \left[z+\int_{0}^{t}-\left(\tilde{r}_{s}+1 / 2\right) d s+\tilde{W}_{t}\right]$. Then, the martingale $\left\{\widetilde{J}\left(t, \tilde{Y}_{t}^{e}\left(e^{z}\right)\right)\right\}$ is a function of the Brownian motion $\left\{\tilde{W}_{t}(z)=z+\tilde{W}_{t}\right\}, \widetilde{J}\left(t, \tilde{Y}_{t}^{e}\left(e^{z}\right)\right)=\Psi\left(t, \tilde{W}_{t}(z)\right)$, where the function $\Psi(t, z)=\widetilde{J}\left(t, e^{-\int_{0}^{t}\left(\tilde{r}_{s}+1 / 2\right) d s} e^{z}\right)$. The martingale property of $\left\{\Psi\left(t, \tilde{W}_{t}(z)\right)\right\}$ implies that $\Psi(t, z)$ is a space-time harmonic solution of the heat equation $\partial_{t} \Psi(t, z)+\frac{1}{2} \Psi_{z z}(t, z)=0$. By the Widder theorem, there exists a positive measure $m$ such that $\Psi(t, z)=\widetilde{J}\left(t, e^{-\int_{0}^{t}\left(\tilde{r}_{s}+1 / 2\right) d s} e^{z}\right)=\int_{\mathbb{R}} e^{\left[(1-\beta) z-\frac{1}{2}(1-\beta)^{2} t\right]} m(d \beta)$, with $\beta>1$. The proof of Theorem 4.3 when $\tilde{\zeta}=1$ is achieved by taking the inverse change of variable $z=\ln (y)+\int_{0}^{u}\left(\tilde{r}_{s}+1 / 2\right) d s$, and $\mu(d \beta)=(\beta-1) m(d \beta)$; the general case is attained after taking the inverse of the change of time. Monotonicity and convexity implies that $\beta>1$.

\subsubsection{Characterization of the primal SPDE solutions}

The primal marginal utility is obtained by inverting the marginal conjugate utility.

INITIAL POWER UTILITY For initial conjugate power utility, the solution is simple since the power utility of a conjugate utility $\tilde{H}_{t}^{(\beta)} \frac{y^{1-\beta}}{\beta-1}$ is a power utility $J^{(\alpha)}(t, x)=H_{t}^{(\alpha)} \frac{x^{1-\alpha}}{1-\alpha}$, where $\alpha=1 / \beta \in(0,1)$ and $H_{t}^{(\alpha)}=\left(\tilde{H}_{t}^{(\beta)}\right)^{\alpha}$. The characteristic process $X_{t}(x)$ is also linear in $x$ and given by $\chi_{t}(x)=x \tilde{H}_{t}^{(\beta)}\left(Y_{t}^{e}\right)^{-\beta}$. In the general aggregate case, the problem is less explicit, but can be formulated as a pure problem of convex analysis: what is the inverse of the mixture of marginal conjugate power functions? Here, the randomness of the problem does not play any role.

The answer is given via an other optimization problem with the sup-convolution of concave functions with initial budget constraint; the main characteristic of the solution is that the derivative of the conjugate is 
the sum of the derivative of the conjugate functions.

We denote by $\nu(d \alpha)$ the Borel measure defined, using the notation $\alpha=1 / \beta$, by $\int_{0}^{1} k(\alpha) \nu(d \alpha)=$ $\int_{1}^{\infty} k(1 / \beta) \mu(d \beta)$. Let us recall the solution of the problem in the deterministic case with easier notations.

Proposition 4.2 (Optimal Pareto allocation problem). Let us consider a family of conjugate power utilities $\left(\tilde{j}^{(\beta)}\right)_{\beta}$ and the conjugate utility $\tilde{j}^{(\mu)}$ which is a mixture of them. Then, the primal utility $j^{(\nu)}(x)$ read along the wealth $x^{(\nu)}(y)=-\tilde{j}_{y}^{(\mu)}(y)$ is still a mixture, and

$$
\left\{\begin{array}{l}
\tilde{j}^{(\mu)}(y)=\int_{1}^{\infty} \tilde{j}^{(\beta)}(y) \mu(d \beta), \quad x^{(\nu)}(y)=\int_{0}^{1} x^{(\alpha)}(y) \nu(d \alpha), \\
j^{(\nu)}\left(x^{(\nu)}(y)\right)=\int_{0}^{1} j^{(\alpha)}\left(x^{(\alpha)}(y)\right) \nu(d \alpha)=\int_{1}^{\infty} \beta \tilde{j}^{(\beta)}(y) \mu(d \beta) .
\end{array}\right.
$$

Furthermore, the utility $j^{(\nu)}$ is the sup-convolution of power utilities $j^{(\alpha)}$

$$
j^{(\nu)}(x)=\sup \left\{\int_{0}^{1} j^{(\alpha)}\left(z^{\alpha}(x)\right) \nu(d \alpha) ; \int_{0}^{1} z^{\alpha}(x) \nu(d \alpha)=x\right\} .
$$

The "Pareto" supremum is achieved at the family $\left\{z^{(\alpha)}(x):=x^{(\alpha)}\left(j_{z}^{(\nu)}(x)\right), \alpha\right\}$.

This proposition can be applied to $\widetilde{J}^{(\mu)}(t, y)$ at any time $t$, but the characteristic process $X_{t}^{(\nu)}$ must satisfy some time-coherency. This constraint is easy to verify since the characteristic processes $X_{t}^{(\alpha)}(x)$ with power primal utility are also linear. Moreover, since the power utility are time separable, the Paretooptimal initial allocation is propagated with the same rule than at time 0 , using an aggregating measure evolving randomly over the time. Indeed, using the notations of the previous Proposition, the first order condition allows to write the equilibrium risky asset as a mixture of linear process.

$$
\text { (Mixing) }\left\{\begin{array}{l}
x_{t}^{(\nu)}\left(x^{(\nu)}(y)\right)=\int_{1}^{\infty} y^{-\beta} \tilde{H}(t, \beta)\left(Y_{t}^{e}\right)^{-\beta} \mu(d \beta)=\int_{1}^{\infty} x^{(\alpha)}(y) X_{t}^{(\alpha)} \nu(d \alpha) \\
J^{(\nu)}\left(t, X_{t}^{(\nu)}\left(x^{(\nu)}(y)\right)\right)=\int_{0}^{1} J^{(\alpha)}\left(t, x^{(\alpha)}(y) X_{t}^{(\alpha)}\right) \nu(d \alpha)
\end{array}\right.
$$

Then we have,

Theorem 4.4. A economic equilibrium holds if and only if there exists a positive Borel measure $\nu$ on $(0,1)$ such that,

(i) The utility process $J^{(\nu)}$ is given as the sup-convolution:

$$
J^{(\nu)}(t, x)=\sup \left\{\int_{0}^{1} J^{(\alpha)}\left(t, z_{\alpha}\right) \nu(d \alpha) ; \int_{0}^{1} z_{\alpha} \nu(d \alpha)=x\right\} .
$$

The supremum is achieved at the family $\left\{x^{\alpha}(t, x):=\left(J_{z}^{(\alpha)}\right)^{-1}\left(t, J_{z}^{(\nu)}(t, x)\right), \alpha\right\}$ satisfying the condition $\int_{0}^{1} x^{\alpha}(t, x) \nu(d \alpha)=x$.

(ii) Economic interpretation Assume the initial wealth optimally Pareto allocated, then at any time the allocation generated by the individual optimal wealth processes $X^{(\alpha)}\left(t, x^{(\alpha)}(x)\right)$ is Pareto optimal for the aggregated utility $J^{(\nu)}\left(t, X_{t}^{(\nu)}(x)\right)$ and the optimal wealth at time $t$.

The proof of this Theorem is given in the Appendix.

Conclusion of the section: The last section has been motivated by different works [Sam38, Sam48, Wan93, DR97, Art99, GR13, CE16 on the neo-classical economic equilibrium theory, whose two pillars 
are the representative agent, and the individual rationality. At the equilibrium (see also Dumas \& al [Dum17]), the best strategy is the technology, whose dynamics is known, and the problem is to find the preferences (utility) of the principal agent, as its utility. But, a little attention is paid to the existence of equilibrium. The He and Leland [HL93] paper considers this question, but only from the viewpoint of the primal problem. They obtain a strongly non linear PDE on the coefficient of $X$, in the Markovian case, and can only give some examples of solutions. Unfortunately, the solution is quasi-trivial since in the Markovian case, the technology is a monotonic function of a geometric Brownian motion. The result is the same in random environment. The Markovian case gives an easy interpretation of the randomness. Assume the randomness generated by a factor $\Theta_{t}$, solution of a SDEs system such that $(X, Y, \Theta)$ admits a unique solution. The assumption is that $\Theta$ can appear in the $X$ coefficient only, but $X$ cannot appear in $\Theta$-coefficients. Then the previous results remain valid provided to look for dynamic utilities which are not functions of the parameter $\Theta$, because the finite variation assumption. In this case, the dynamic utility at time $t$ will depend on the entire past of $\Theta_{s} ; s \leq t$ through the processes $X$ and $Y$.

\subsection{Conclusion}

In this work we have provided a necessary and sufficient condition for the existence of a solution to the general problem of revealed utility, using very basic tools of analysis and the theory of integration. We have made almost no assumptions about the regularity of the processes in time $t$ and the constructed utilities are only differentiable in $x$. To be aware of the efficiency of our method and the extent of our results, it is enough to refer to existing work in the semimartingale framework where calculations are tedious and assumptions are numerous. Requiring to treat the problem in an abstract way has clarified many subtleties including the role of the initial conditions and how to deal with the Stieltjes integral near to zero. The different notions of orthogonality introduced for the first time in this type of problem are the keys of this work. These difficulties are particularly well-illustrated in the Markovian economic equilibrium problem. By approaching the problem from the conjugate point of view to be concentrated on the pricing kernel process only (as in the first part) and exploiting the necessary and sufficient orthogonality condition of our main result yields to the complete resolution of this equilibrium problem, until now without a satisfactory answer.

Also, this orthogonality conditions undoubtedly plays an important role in Markov framework with factor $\Theta$, because they give us the necessary and sufficient conditions in the form of PDEs. Solving them is still an open question that will be studied in a forthcoming paper. Finally, since we have no regularity assumptions with respect to the time, our results can be applied in the discrete frame, and in different settings, as preference learning in which the goal is to learn a predictive preference model from observed preference information.

\section{Appendix}

Proof of Theorem 4.4 Using the notation $x^{(\nu)}(y)=-\tilde{j}_{y}^{(\mu)}(y)=x$ in equation (4.18), follows

$$
J^{(\nu)}\left(t, X_{t}^{(\nu)}(x)\right)=\int_{0}^{1} J^{(\alpha)}\left(t, x^{(\alpha)}\left(j_{z}^{(\nu)}(x)\right) X_{t}^{(\alpha)}\right) \nu(d \alpha) .
$$

As $X_{t}^{(\nu)}(x)=-\widetilde{J}_{y}^{(\mu)}\left(t, j_{z}^{(\nu)}(x) Y_{t}\right)$, it is a monotonic function in $x$ with inverse $\left(j_{z}^{(\nu)}\right)^{-1}\left(J_{z}^{(\nu)}(t, x) / Y_{t}\right)$, we obtain

$$
J^{(\nu)}(t, x)=\int_{0}^{1} J^{(\alpha)}\left(t, x^{(\alpha)}\left(J_{z}^{(\nu)}(t, x) / Y_{t}\right) X_{t}^{(\alpha)}\right) \nu(d \alpha) .
$$


Now, as $x^{(\alpha)}(y) X_{t}^{(\alpha)}=-\widetilde{U}_{y}^{\beta}\left(t, y Y_{t}\right)(\beta=1 / \alpha)$, the quantity $x^{(\alpha)}\left(J_{z}^{(\nu)}(t, x) / Y_{t}\right) X_{t}^{(\alpha)}$ is equal to $-\widetilde{J}_{y}^{\beta}\left(t, J_{z}^{(\nu)}(t, x)\right)$. In other words,

$$
J^{(\nu)}(t, x)=\int_{0}^{1} J^{(\alpha)}\left(t,-\widetilde{J}_{y}^{\beta}\left(t, J_{z}^{(\nu)}(t, x)\right)\right) \nu(d \alpha) .
$$

Moreover, identical reasoning as in the proof of the previous result, using the inequality

$$
J^{(\alpha)}\left(t,-\widetilde{J}_{y}^{\beta}(t, y)\right) \geq J^{(\alpha)}\left(t, z_{\alpha}\right)+\left(\widetilde{J}_{y}^{\beta}(t, y)-z_{\alpha}\right) y, \forall z_{\alpha}>0,
$$

integrating and replacing $y$ by $J_{z}^{(\nu)}(t, x)$, follows

$$
J^{(\nu)}(t, x) \geq \sup \left\{\int_{0}^{1} J^{(\alpha)}\left(t, z_{\alpha}\right) \nu(d \alpha)+J_{z}^{(\nu)}(t, x) \int_{0}^{1}\left(\widetilde{J}_{y}^{\beta}\left(t, J_{z}^{(\nu)}(t, x)\right)-z_{\alpha}\right) \nu(d \alpha)\right\},
$$

with equality iff $\int_{0}^{1} \widetilde{J}_{y}^{1 / \alpha}\left(t, J_{z}^{(\nu)}(t, x)\right) \nu(d \alpha)=\int_{0}^{1} z_{\alpha} \nu(d \alpha)$, in this case the supremum is achieved at $z_{\alpha}=$ $-\widetilde{J}_{y}^{\beta}\left(t, J_{z}^{(\nu)}(t, x)\right)=\left(J_{z}^{(\alpha)}\right)^{-1}\left(t, J_{z}^{(\nu)}(t, x)\right)$. But, using the definition of $\widetilde{J}^{(\mu)}$ as a mixture, one can observes that the integral $\int_{0}^{1} \widetilde{J}_{y}^{1 / \alpha}\left(t, J_{z}^{(\nu)}(t, x)\right) \nu(d \alpha)$ is equal to $x$. This achieves the proof.

\section{References}

[Art99] W.B. Arthur. Complexity and the economy. Science, 284(5411):107-109, 1999.

[Bac00] Louis Bachelier. Théorie de la spéculation. In Annales scientifiques de l'École normale supérieure, volume 17, pages 21-86, 1900.

[BEK05] P. Barrieu and N. El Karoui. Inf-convolution of risk measures and optimal risk transfer. Finance and stochastics, 9(2):269-298, 2005.

[BRT09] F. Berrier, L. Rogers, and M. Tehranchi. A characterization of forward utility functions. Preprint, 2009.

[CE16] C.P. Chambers and F. Echenique. Revealed preference theory, volume 56. Cambridge University Press, 2016.

[CKO01] U. Chajewska, D. Koller, and D. Ormoneit. Learning an agent's utility function by observing behavior. In ICML, pages 35-42, 2001.

[DR97] P.H. Dybvig and L.C.G. Rogers. Recovery of preferences from observed wealth in a single realization. The Review of Financial Studies, 10(1):151-174, 1997.

[Dum17] E. Dumas, B.and Luciano. The Economics of Continuous-Time Finance. MIT Press, 2017.

[EKHM17] N. El Karoui, C. Hillairet, and M. Mrad. Construction of an aggregate consistent utility, without pareto optimality. application to long-term yield curve modeling. In International Symposium on BSDEs, pages 169-199. Springer, 2017.

[EKHM18] N. El Karoui, C. Hillairet, and M. Mrad. Consistent utility of investment and consumption: a forward/backward spde viewpoint. Stochastics, 90(6):927-954, 2018.

[EKM13] N. El Karoui and M. Mrad. An Exact connection between two Solvable SDEs and a Non Linear Utility Stochastic PDEs. SIAM Journal on Financial Mathematics, 4(1):697-736, 2013.

[EKPY12] N. El Karoui, E. Pardoux, and M. Yor. In Stochastic Filtering at Saint Flour, Probability at Saint Flour, chapter Les aspects probabilistes du contrôle stochastique, pages 43-203. Springer, Berlin, 2012. 
[FH11] J. Fürnkranz and E. Hüllermeier. Preference learning. Springer, 2011.

[FS03] C. Friedman and S. Sandow. Learning probabilistic models: An expected utility maximization approach. Journal of Machine Learning Research, 4:257-291, 2003.

[FS16] C. Friedman and S. Sandow. Utility-based learning from data. CRC Press, 2016.

[FSS06] J. Fürnkranz, T. Scheffer, and M. Spiliopoulou. Machine Learning: ECML 2006: 17th European Conference on Machine Learning, Berlin, Germany, September 18-22, 2006, Proceedings, volume 4212. Springer Science \& Business Media, 2006.

[GEKR95] H. Geman, N. El Karoui, and J-C. Rochet. Changes of numeraire, changes of probability measure and option pricing. Journal of Applied probability, pages 443-458, 1995.

[GM18] E. Gobet and M. Mrad. Convergence rate of strong approximations of compound random maps, application to spdes. Discrete $\&$ Continuous Dynamical Systems-B, 23(10):4455, 2018.

[GR13] J. Gomez-Ramirez. Don't blame the economists. it is an inverse problem! European JOURNAL of Futures Research, 1(1):1, 2013.

[HH07] V. Henderson and D. Hobson. Horizon-unbiased utility functions. Stochastic Process. Appl., 117(11):1621-1641, 2007.

[Hib12] B. Hibbard. Model-based utility functions. Journal of Artificial General Intelligence, 3(1):1$24,2012$.

[HL93] H. He and H. Leland. On equilibrium asset price processes. The Review of Financial Studies, 6(3):593-617, 1993.

[Kun97] H. Kunita. Stochastic flows and stochastic differential equations, volume 24 of Cambridge Studies in Advanced Mathematics. Cambridge University Press, 1997.

[LT11] S. Lasaulce and H. Tembine. Game theory and learning for wireless networks: fundamentals and applications. Academic Press, 2011.

[MZ03] M. Musiela and T. Zariphopoulou. Backward and forward utilities and the associated pricing systems: The case study of the binomial model. Preprint, 2003.

[MZ10a] M. Musiela and T. Zariphopoulou. Portfolio choice under space-time monotone performance criteria. SIAM Journal on Financial Mathematics, 1(1):326-365, 2010.

[MZ10b] M. Musiela and T. Zariphopoulou. Stochastic partial differential equations and portfolio choice. pages 195-216, 2010.

[NJ04] T.D. Nielsen and F. V. Jensen. Learning a decision maker's utility function from (possibly) inconsistent behavior. Artificial Intelligence, 160(1):53-78, 2004.

[QXL14] Y. Qi, M. Xu, and J. Lafferty. Learning high-dimensional concave utility functions for discrete choice models. Cited on, page 26, 2014.

[Sam38] P.A. Samuelson. A note on the pure theory of consumer's behavior. Economica, 5(17):61-71, 1938.

[Sam48] P.A. Samuelson. Consumption theory in terms of revealed preference. Economica, 15(60):243$253,1948$.

[Sta04] A. Stahl. Approximation of utility functions by learning similarity measures. In Logic versus Approximation, pages 150-172. Springer, 2004.

[Ven65] A.D. Ventzel. On equations of the theory of conditional Markov. Theory of Probability and its Applications, 10:357-361, 1965. 
[Wan93] S. Wang. The local recoverability of risk aversion and intertemporal substitution. Journal of Economic Theory, 59(2):333-363, 1993.

[WTKD04] E.W. William, G. Tesauro, J. O. Kephart, and R. Das. Utility functions in autonomic systems. In 1st International Conference on Autonomic Computing (ICAC 2004), 17-19 May 2004, New York, NY, USA, pages 70-77, 2004.

[WZ11] J. Wang and Y. Zhang. Utilizing marginal net utility for recommendation in e-commerce. pages 1003-1012, 012011. 